\title{
Nanostructured silicon for studying fundamental aspects of nanomechanics
}

\author{
R H Blick ${ }^{1}$, A Erbe, L Pescini, A Kraus, D V Scheible, F W Beil, \\ E Hoehberger, A Hoerner, J Kirschbaum, H Lorenz and J P Kotthaus \\ Center for NanoScience, Ludwig-Maximilians-Universität, Geschwister-Scholl-Platz 1, \\ 80539 München, Germany \\ E-mail: robert@nanomachines.com
}

\begin{abstract}
Nanomechanics features three-dimensional nanostructuring, which allows full exploitation of the mechanical degree of freedom on the nanometre scale. In this work a number of exemplifying experiments on nano-electromechanical systems realized in silicon materials will be presented. First an introduction to the underlying mechanics will be given and finite element methods required for simulations will be discussed. Further topics presented include measurement methods for probing the mechanical properties of free standing nanowires, sensor applications and nonlinear properties of nanomechanical resonators. Other applications such as parametric frequency tuning are demonstrated and the major sources of dissipation are discussed. Finally, an outlook over the fundamental limits of nanoresonators is given.
\end{abstract}

\section{Contents}

1. Introduction 906

2. Modelling nanomechanical systems 906

2.1. The Duffing equation 907

2.2. Finite element simulations 910

3. Sample fabrication $\quad 912$

4. Experimental techniques 913

5. Sensor applications $\quad 915$

6. NEMS in the nonlinear regime $\quad 919$

7. Parametric amplifier $\quad 926$

1 Author to whom any correspondence should be addressed. 
8. Dissipation 932

8.1. Ohmic losses 932

8.2. Viscous or external losses 934

8.3. Internal losses 935

8.4. Electronic environment 936

9. Quantum-limited displacement detection 937

$\begin{array}{lr}\text { 10. Outlook } & 942\end{array}$

Acknowledgments $\quad 942$

References $\quad 943$

\section{Introduction}

The use of mechanical devices dates back quite some time considering for example the plough ${ }^{2}$. Tools like that are now reinvented on the nanometre scale, e.g. the so-called nanoplough, being used for 'mechanical' lithography [1]. Of course mechanics on the nanometre scale is very different to the macroscopic version. The difficulties arising are mostly caused by the different forces of importance on this scale: gravity is not dominant when considering its influence on a 'bridge' with a width of only $20 \mathrm{~nm}$. The much more relevant forces are clearly found in surface tension or the van der Waals interaction.

Apart from the interest in basic research to gain control of the nanometre world, the development of the appropriate nano-electromechanical tools is indeed important for industry, a clear advantage being the convergence of processing techniques for integrated silicon circuits and semiconductor nano-electromechanical systems (NEMS). The key element of all NEMS is the doubly clamped string resonator. Hence, this rather simple device was chosen to elucidate the basic physics of NEMS. The first aim in designing and building such resonators is complete control of the mechanical quality factor $Q$. In the following the straightforward definition $Q=f_{0} / f_{b}$ is used, where $f_{0}$ is the eigenfrequency and $f_{b}$ the full width at half maximum at resonance. Controlling $Q$, pushing eigenfrequencies above the $\mathrm{GHz}$ barrier, understanding the dissipative mechanisms and eventually reaching the realm of quantum mechanical mechanics are the essential goals in developing NEMS circuits.

The different sources of dissipation are to be studied in detail, starting from internal dissipation mechanisms due to dislocation and impurities over to external sources such as losses due to clamping points and adsorbed layers of water or chemicals. So far, only the very basic properties have been addressed. At the same time another important technical factor has to be brought into the discussion, namely the control of bandwidth of the mechanical device under study. The tunability of both $Q$ and bandwidth, the eigenfrequency $f_{0}$ and the degree of coupling of such nanomechanical systems are of prime importance. Apart from the regime of linear response, nanomechanical resonators are the perfect tool for investigating mechanical nanosystems in the chaotic regime.

\section{Modelling nanomechanical systems}

The simplest mechanical resonator usually considered is a doubly clamped beam, comparable to a violin or guitar string [2]. In the following the governing equation for such a beam-the Duffing equation - is considered in some detail, since from this most of the basic properties of a mechanical resonator can be derived. Moreover, trying to model the mechanical properties of more complex resonator geometries is hardly possible analytically. This can be accomplished

2 The first ploughs are depicted on small clay plaques found at Uruk IV in Iraq, dated around 3200 BC. 
using finite element methods (FEMs) as will be described below in section 2.2. For a more detailed description of the mathematics involved the textbooks by Landau and Lifshitz [3] and by Kinsler et al [4] are recommended.

\subsection{The Duffing equation}

The differential equation for the deflection $u(z)$ is found to be

$$
E \Theta \mathrm{d}^{4} u(z)=-F(z)
$$

where $E$ is Young's modulus, $\Theta$ the moment of inertia, $F$ the applied force and $z$ gives the distance to the clamping points. As an example a force $W$ is considered, acting at the point $z=L$, while the bar is clamped at $z=0: F(z)=W \delta(z-L)$. From equation (1) it is found after integration that

$$
E \Theta \frac{\mathrm{d}^{3} u(z)}{\mathrm{d} z^{3}}=W, \quad 0<z<L .
$$

Another integration and using the fact that the torque $\mathrm{d}^{2} u(z) / \mathrm{d} z^{2}=0$ at $z=L$ leads to

$$
E \Theta \frac{\mathrm{d}^{2} u(z)}{\mathrm{d} z^{2}}=W(z-L) .
$$

Another two integrations and considering $z=0$ and $\mathrm{d} u / \mathrm{d} z=0$ we find

$$
E \Theta \frac{\mathrm{d} u(z)}{\mathrm{d} z}={ }_{2}^{1} W z^{2}-W L z,
$$

and

$$
E \Theta u(z)={ }_{6}^{1} W z^{3}-{ }_{2}^{1} W L z^{2} .
$$

The deflection at $z=L$ is $u(L)=-W L^{3} /(3 E I)$ and the angle is

$$
\theta=\begin{gathered}
\mathrm{d} u(z) \\
\mathrm{d} z
\end{gathered} \|_{z=L}=\begin{gathered}
3 u(L) \\
2 L
\end{gathered} .
$$

Extending now equation (1) by applying an external force and using Newton's second law, the equation of motion of the beam is

$$
E \Theta \frac{\mathrm{d}^{4} u(z, t)}{\mathrm{d} z^{4}}=-\rho S^{\mathrm{d}^{2} u(z, t)} \begin{gathered}
\mathrm{d} t^{2}
\end{gathered},
$$

where $\rho$ is the density of the material and the strain is defined by $S=\Sigma F$ with stress $\Sigma$ and force $F$. For sinusoidal vibrations, $u(z, t)=u(z) \cos (\omega t+\alpha)$, the equation can be written in the form

$$
E \Theta \frac{\mathrm{d}^{4} u(z, t)}{\mathrm{d} z^{4}}=-\rho S \omega^{2} u(z) .
$$

Introducing $\kappa^{4}=\left(\omega^{2} \rho S\right) /(E \Theta)$ the general solution is

$$
u(z)=A \cos \kappa z+B \sin \kappa z+C \cosh \kappa z+D \sinh \kappa z,
$$

with $A, B, C, D$ as parameters. If one end of the beam is clamped and the other one is free to move the boundary conditions at $z=0$ are $u=0$ and $\mathrm{d} u / \mathrm{d} z=0$, and at the free end $z=L$ the vertical force and torque vanish, $\mathrm{d}^{2} u / \mathrm{d} z^{2}=0$ and $\mathrm{d}^{3} u / \mathrm{d} z^{3}=0$. This finally gives

$$
\cos \kappa L \cosh \kappa L+1=0
$$


with which the lowest mechanical mode is found to be at $\kappa L \cong 1.87$ with a frequency of

$$
f=\stackrel{0.56}{L^{2}} / \begin{gathered}
E \Theta \\
\rho S
\end{gathered} .
$$

Considering now a harmonic wave equation to model a one-dimensional resonator by including a term of third order. This yields the Duffing equation (see [5]):

$$
\ddot{x}+2 \mu \dot{x}+\omega_{0}^{2} x+k_{3} x^{3}=F \cos \omega t,
$$

where $\mu$ is the attenuation constant, $\omega_{0}$ the eigenfrequency and $F$ the strength of the driving force. The constant $k_{3}$ determines the degree of nonlinearity. In the limit of small displacement the nonlinear term can be omitted and we obtain the differential equation of a harmonic oscillator:

$$
\ddot{x}+2 \mu \dot{x}+\omega_{0}^{2} x=F \cos \omega t .
$$

The solution for this equation is given by

$$
x(t)=a \exp (-\mu t) \cos \left[\sqrt{\left.\omega_{0}^{2}-4 \mu^{2} t+\beta\right]+} \underset{\sqrt{ }\left(\omega_{0}^{2}-\omega^{2}\right)^{2}+4 \mu^{2} \omega^{2}}{\cos (\omega t+\varphi) .}\right.
$$

The first term gives the homogeneous solution, while for $t \gg \mu^{-1}$ only the second term of equation (14) remains with the stationary solution

$$
x(t)=\frac{F}{\sqrt{\left(\omega_{0}^{2}-\omega^{2}\right)^{2}+4 \mu^{2} \omega^{2}}} \cos (\omega t+\varphi) .
$$

The phase difference $\varphi$ of excitation and response is given by

$$
\varphi=\arctan \left(\begin{array}{c}
2 \mu \omega \\
\omega^{2}-\omega_{0}^{2}
\end{array}\right),
$$

while the maximal amplitude $x_{0, \max }$ is

$$
x_{0, \max }=\begin{gathered}
F \\
2 \mu \omega_{0}
\end{gathered} .
$$

A possible way to solve equation (12) is obtained by the harmonic balance method (see [7]). This implies that the stationary solutions can be approximated by

$$
x(t)=x_{0} \cos (\omega t+\varphi)
$$

The nonlinear term is then replaced by a linear one depending on the amplitude $k_{3} x^{3} \mapsto$ $k^{*}\left(x_{0}\right) x$. Starting with

$$
\begin{gathered}
x(t)^{3}=\left[x_{0} \cos (\omega t+\varphi)\right]^{3}=x_{0}^{3}\left[{ }_{4}^{3} \cos (\omega t+\varphi)+{ }_{4}^{1} \cos 3(\omega t+\varphi)\right] \\
={ }_{4}^{3 x_{0}^{2}} x(t)+{ }_{4}^{x_{0}^{3}} \cos 3(\omega t+\varphi)
\end{gathered}
$$

and neglecting the term ${ }_{4}^{x_{0}^{3}} \cos 3(\omega t+\varphi)$, a linear equation is found,

$$
\ddot{x}+2 \mu \dot{x}+\left(\omega_{0}^{2}+{ }_{4}^{3 k_{3}} x_{0}^{2}\right) x=F \cos \omega t .
$$

Amplitude and phase of the stationary solution (equation (18)) follow with equations (15) and (16)

$$
x_{0}=\frac{F}{\sqrt{\left(\omega_{0}^{2}+{ }_{4}^{3 k_{3}} x_{0}^{2}-\omega^{2}\right)^{2}+4 \mu^{2} \omega^{2}}}
$$


and

$$
\varphi=\arctan \left(\begin{array}{c}
2 \mu \omega \\
\omega^{2}-\left(\omega_{0}^{2}+{ }_{4}^{3 k_{3}} x_{0}^{2}\right)
\end{array}\right) .
$$

Equation (21) allows determination of the resonance amplitude $x_{0}$. The square value of equation (21) leads to an algebraic expression of third order in $x_{0}^{2}$

$$
x_{0}^{2}\left[\left(\omega_{0}^{2}-\omega^{2}+\frac{3 k_{3}}{4} x_{0}^{2}\right)^{2}+4 \mu^{2} \omega^{2}\right]=F^{2},
$$

where the real part of the solution yields the resonance amplitudes. Focusing on the interval of the resonance, i.e. $\left|\omega-\omega_{0}\right| \ll \omega_{0}$, the following approximation in equation (23) is valid: $\omega_{0}^{2}-\omega^{2}=\left(\omega_{0}-\omega\right)\left(\omega_{0}+\omega\right) \approx 2 \omega_{0}\left(\omega_{0}-\omega\right)$ and $\omega^{2} \approx \omega_{0}^{2}$. This finally gives the desired equation

$$
x_{0}^{2}\left[\left(\omega_{0}-\omega+\frac{3 k_{3}}{8 \omega_{0}} x_{0}^{2}\right)^{2}+\mu^{2}\right] \approx\left(\begin{array}{c}
F \\
2 \omega_{0}
\end{array}\right)^{2} .
$$

Solving for $\omega-\omega_{0}$ results in

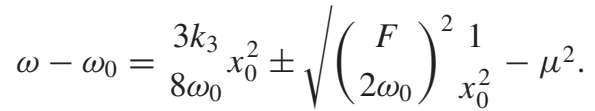

This equation assigns each value $x_{0}$ a specific number of frequencies depending on

$$
x_{0} \leqslant \underset{2 \mu \omega_{0}}{F} .
$$

This relation allows us to find the deviation of the resonance frequency from the regime of linear response

$$
x_{0, \max }=\begin{gathered}
F \\
2 \mu \omega_{0}
\end{gathered}
$$

and

$$
\omega_{\max }-\omega_{0}=\begin{gathered}
3 k_{3} \\
8 \omega_{0}
\end{gathered}\left(\begin{array}{c}
F \\
2 \mu \omega_{0}
\end{array}\right)^{2} .
$$

The maximal amplitude is identical to the linear case, see equation (17). In figure 1 resonance traces are given following equation (25) for a variety of values for $F$. The parameters were taken from the samples measured.

From figure 1 it is seen that at certain amplitudes $F$ of the external excitation, regions of multiply defined frequencies are found. The transition into this regime occurs at the so-called critical amplitude $F_{c}$ of the driving force, corresponding to a critical displacement amplitude $x_{c}$. The relation connecting the displacement $x_{c}$ with the resonator's geometry is [6]

$$
x_{c}=\frac{2 b}{\sqrt{{ }_{2}^{1}} Q\left(1-v^{2}\right)},
$$

where $Q$ is the mechanical quality factor of the resonator, $b$ is the width and $v$ is the lateral extension coefficient of the material. Usage of this critical point is extremely important for application of NEMS in sensor technology (see section 5). In the bistable region beyond the critical frequency three different solutions of the wave equation are found and the resonator shows a pronounced hysteresis. 


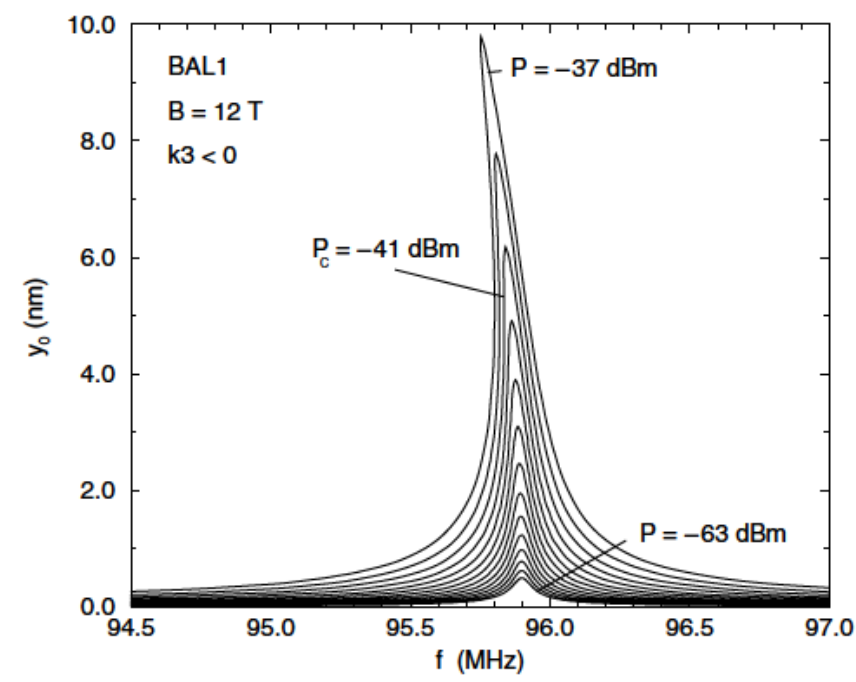

Figure 1. Nonlinear resonance trace for increasing values of $F$ in equation (25), indicated by the power level $P$.
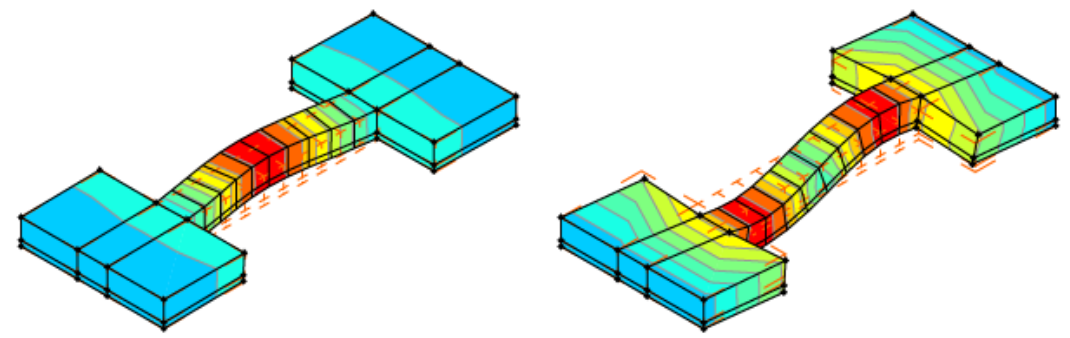

Figure 2. Modes of a doubly clamped nanobeam: (a) basic and (b) excited mode.

\subsection{Finite element simulations}

In order to gain a deeper understanding of the mechanical and electro-magnetic properties of the nanoresonators, FEMs have to be employed, since only simple geometries can be modelled analytically [8]. The underpinning idea of these simulations is to achieve a quantitative comparison with the nanoscale mechanical device. However, it turned out that the accuracy with which a predicted eigenfrequency is obtained in the measurement is of the order of $10 \%$. The electro-magnetic problem solvers on the other hand give quite reliable values regarding impedance matching of the whole circuit. Hence, the design process of a resonator is determined by a close inspection of its properties first in the simulators and a number of iterative steps ensuring the best match to the specifications [9].

The program used for simulations of the mechanical properties ${ }^{3}$, Solvia ${ }^{\oplus}$, allows determination of the effective strain caused by an external stress in three dimensions. A metallic top layer can be included as well as different geometries of the structural clamping points. In figure 2(a) the simplest $\lambda / 2$-mode is shown in a three-dimensional representation, while in figure 2(b) a more complex excited mode of the system is depicted. Moreover, harmonics, sub- and ultra-harmonics of the mechanical oscillation can be reproduced. The

${ }^{3}$ Solvia, finite element solver for mechanical modelling, v. 95.2. 


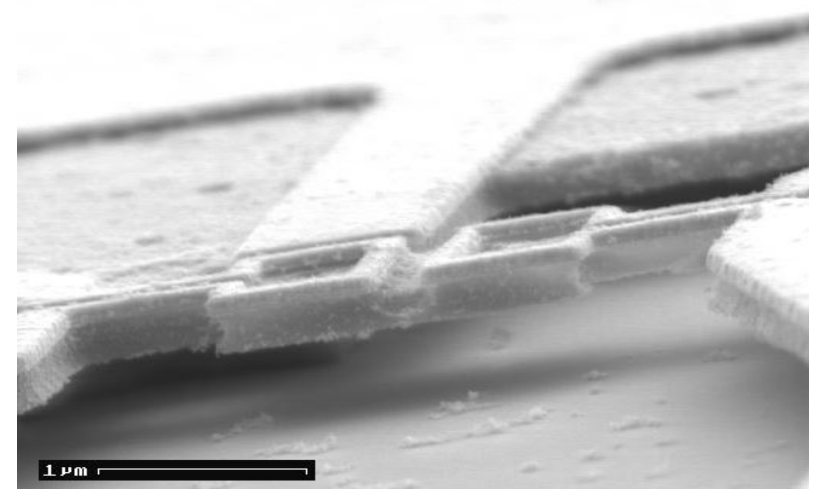

Figure 3. Torsional resonator with a drive and a detection metal loop on top.

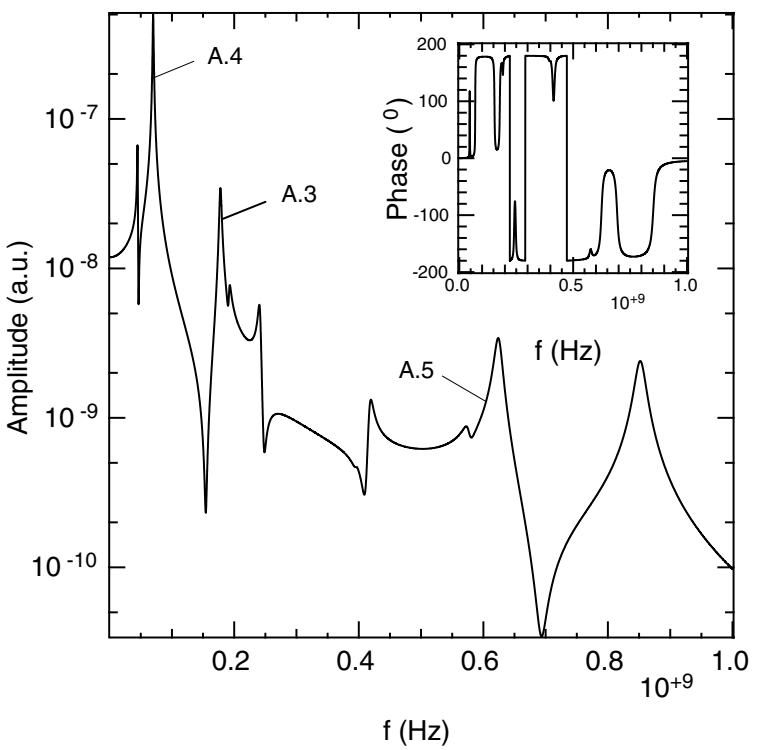

Figure 4. Calculated mode spectrum of a singly clamped nanobeam in a plot of amplitude versus frequency (from [10]).

modes in these simple beam resonators are finally used to gather information for the comparison to more complex resonators like the torsional cradle (see figure 3). In addition to calculating the individual mode shapes the program also allows us to obtain the frequency dependence of the displacement amplitude as shown for one of the singly clamped resonators in figure 4 .

For sensor applications active $Q$-tuning is highly desirable. For such purposes different geometries can be investigated as shown in figure 5, which support a number of gating electrodes similar to quantum dots for biasing the resonator. In this way the potential can be changed strongly, depending on the specific mechanical mode and on gate geometry. Additionally, such a layout with a number of electrodes enables us to parametrically pump either the central part of the resonator or the fins on the left and right. In this way the whole setup is much more versatile and the mechanical spectrum is controllable. 


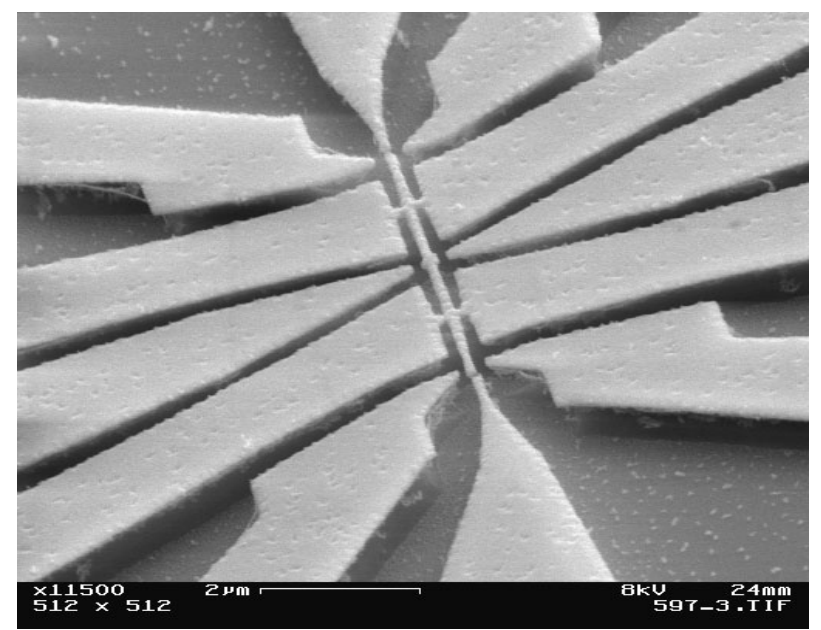

Figure 5. Advanced resonator layout, including multiple tuning gates and 'quality fins' for active $Q$-tuning [9].

In a similar fashion the electromagnetic environment of the resonators is determined by numerical methods. The programs applied here $\operatorname{are}^{4}$ Sonnet $^{\oplus}$ and $^{5}$ MAFIA ${ }^{\odot}$. While Sonnet gives reliable results in the range of $10 \mathrm{MHz}-500 \mathrm{GHz}$, MAFIA covers the whole frequency range from dc on to the far infrared. In figure 6 a top view of a singly clamped beam is shown calculated: the electric field distribution between a gating electrode and the tip of the clapper is calculated by MAFIA ${ }^{\odot}$ when a voltage of $V_{g}=1 \mathrm{~V}$ is applied according to $E=C V_{g}^{2} / 2$, where $E$ is the electric field strength. The capacitance between gate and tip for the case of interest is found to be $C=21 \mathrm{aF}$.

\section{Sample fabrication}

Fabrication of the devices presented in this work is performed in clean rooms by semiconductor processing techniques. The materials used are silicon-on-insulator (SOI) wafers. The overwhelming strength of semiconductors is the ultimate purity and precision with which samples can be processed on a large scale. The standard tool for semiconductor structuring is optical lithography.

The two-dimensional patterning and structuring of semiconductors can be extended to build nanostructures in three dimensions. A variety of etching methods is applied to effectively suspend nanostructures, which introduces the mechanical degree of freedom. For threedimensional nanostructures a specific sacrificial layer has to be added to the layer sequence. For silicon we use commercially available SOI substrates with thicknesses of the Si layer and the $\mathrm{SiO}_{2}$ sacrificial layer of 50-200 and 100-400 nm [11], respectively (Smart-Cut wafers) [12]. After definition of a nanomechanical resonator by electron beam lithography, the masked sample is dry etched in a reactive-ion etcher in order to obtain the desired mesa structure with clear-cut walls [13]. Finally, a hydrofluoric wet etch is performed removing the $\mathrm{SiO}_{2}$ sacrificial layer below the resonators and the metallic etch mask leading to a suspended bridge as shown in figure $7[14,15]$. Apparently the whole top layer can also be suspended and attached to a material of choice [17].

4 Sonnet, electromagnetic finite element solver, v. 6.0.

5 MAFIA, electromagnetic finite element program, v. 3.20. 


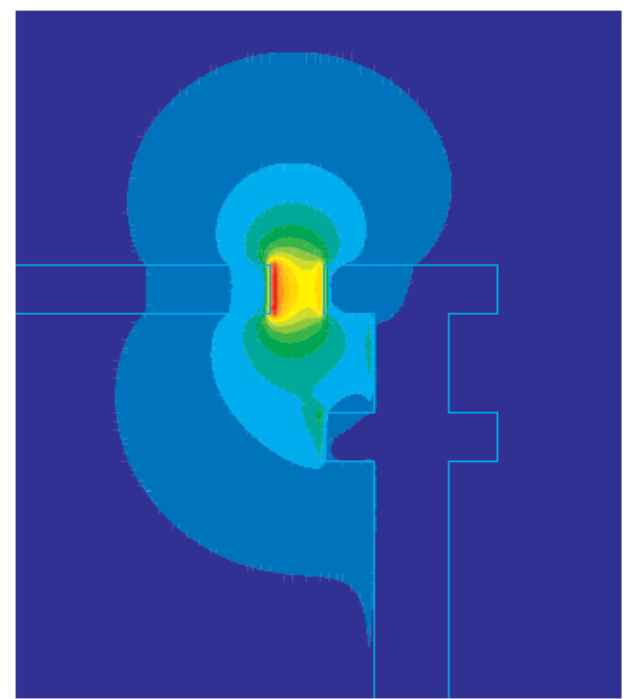

Figure 6. Electric field distribution between a gate electrode and the tip of a nanomechanical resonator (from [10]).

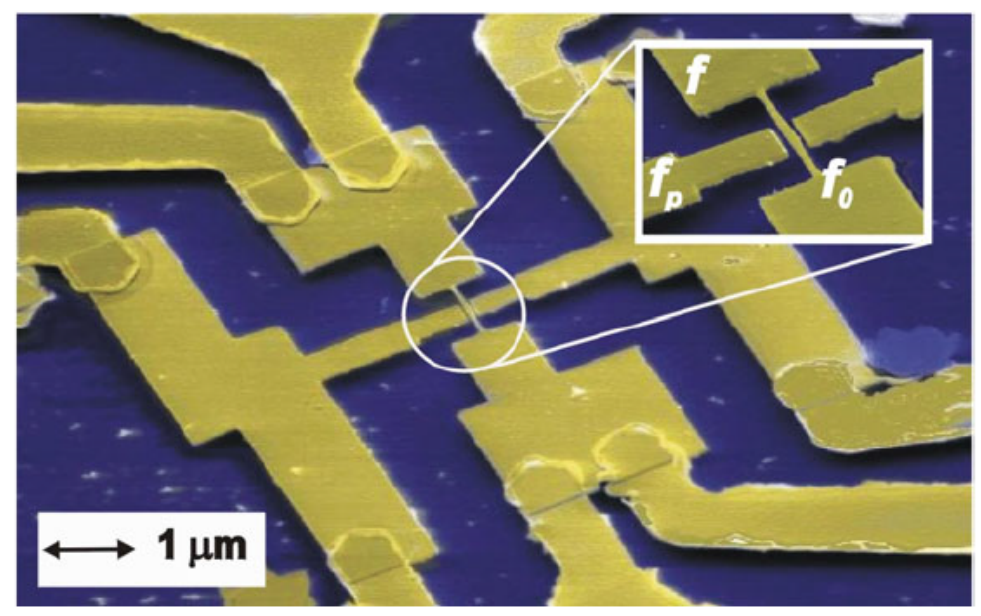

Figure 7. Scanning electron beam micrograph of one of the electromechanical resonators used. The silicon beam is covered by a thin Au sheet of $50 \mathrm{~nm}$, which allows for impedance matching to the circuitry and coupling of the driving frequency $f$ to the mechanical eigenfrequency $f_{0}$. The electrodes on the left and right enable application of an additional dc bias and/or ac modulation at a pump frequency $f_{p}$. The inset shows a magnification of the nanobridge.

\section{Experimental techniques}

In this section a brief survey is given of the techniques at hand for probing the mechanical properties of nanomechanical systems. The common aim of the various probing mechanisms is to achieve high speed operation and a high sensitivity in displacement detection. In all setups probing nano-electromechanical circuits it has to be considered that excitation as well as detection always require electronic coupling. Most common are two techniques as electronic excitation mechanisms: magneto-motive and capacitive excitation. 


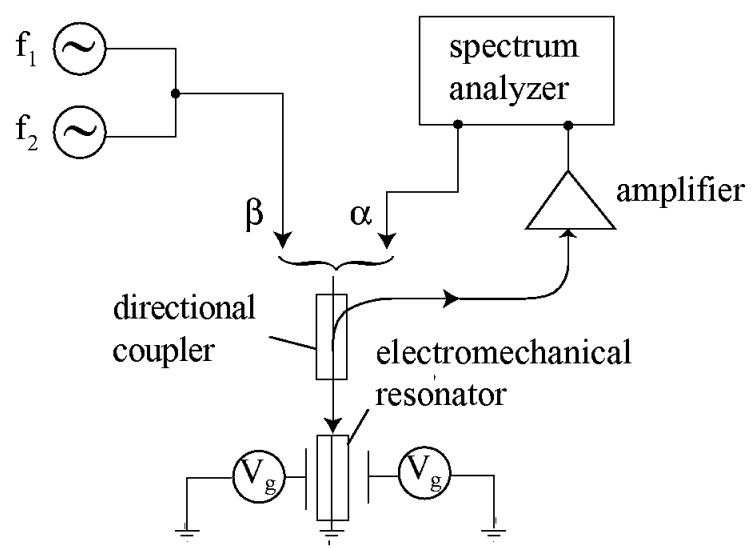

Figure 8. Experimental setup for sampling the properties of mechanical nanoresonators: a circuit diagram of the spectrum/network analyser is employed scanning the frequency range of interest and the reflected signal is measured directly $(\alpha)$, while mechanical mixing can be analysed by combining two synthesizers $\left(f_{1}, f_{2}\right)$ and detecting the reflected power $(\beta)$.

The simplest one is magneto-motive excitation, where a Lorentz force is generated by placing the metallized nanobeam in an external dc magnetic field and sending an alternating current along the length of the conducting beam [18-20]. The motion in the magnetic field in turn induces an electromagnetic force in the suspended wire, which can be read out. This mechanism is very reliable and has been extensively used throughout this work. A certain disadvantage for applications obviously is the need to apply magnetic fields of the order of $B>2 \mathrm{~T}$. Also, heating by the excitation current influences the mechanical properties of the nanobeam, since dissipation on this size scale is strongly altered.

A typical setup for magneto-motive excitation is shown in figure 8: the displacement of the oscillating motion causes a change in the amplitude dependent impedance $\hat{Z}_{\text {res }}$ of the resonator. The total impedance of the beam resonator in a magnetic field is given as

$$
\hat{Z}_{\text {res }}(\omega)=R+\mathrm{i} \omega A(\omega){ }_{m_{\mathrm{eff}}}^{L^{2} B^{2}} \exp (\mathrm{i} \phi),
$$

where $m_{\text {eff }}$ is the effective mass of the beam and the length of the beam $l$ is connected via $L=l \pi / 2$, while $A(\omega)$ gives the absolute value of the dynamic susceptibility and $\phi$ the corresponding phase. This can be simplified under a harmonic excitation at the eigenfrequency $\omega_{0}=2 \pi f_{0}$ to

$$
\hat{Z}_{\text {res }}\left(\omega=\omega_{0}\right)=R+\frac{L^{2} B^{2}}{2 \mu m_{\mathrm{eff}}},
$$

where $\mu$ is the attenuation constant. Commonly the total dc resistance of the samples is of the order of $R \sim 40-50 \Omega$. The change in impedance is detected by tracing the reflected power using a spectrum/network analyser $(\operatorname{setup} \alpha)$. For further experiments regarding mechanical mixing, harmonic generation, and parametric amplification additional synthesizers can be integrated $(\operatorname{setup} \beta)$.

Most measurements are conducted at $4.2 \mathrm{~K}$ in a sample holder with a residual ${ }^{4} \mathrm{He}$-gas pressure of about $10 \mathrm{mbar}$. This ensures thermal coupling, while it naturally reduces the $Q$. The sample is mounted between interconnecting microstrip lines, designed to feed the circuit with frequencies up to $10 \mathrm{GHz}$, and a magnetic field is applied perpendicular to the beam. For excitation and final amplification we use either a spectrum or a network analyser. 
The hysteresis of the mechanical resonator is probed with an additional synthesizer, which can be ramped from lower to higher frequencies and vice versa. Capacitive excitation relies on sufficiently large coupling of the gate electrodes to the nanomechanical resonator under test. The main limitation is given by the large electric field strength of the order of $10^{5}-10^{8} \mathrm{~V} \mathrm{~m}^{-1}$ across two contacts. This eventually leads to field emission and a degradation of the gates.

On the detector side several methods can be realized for highly sensitive displacement detection. The method featured in this work is a capacitive transducer. Moreover, optical detection can also be used [21], which is not pursued in this work. As mentioned above the change in impedance can either be detected directly by tracing the reflected power using a network analyser in combination with a scattering parameter testset or by capacitive coupling of the aforementioned sidegates. The preamplifier employed is a low-noise broad-band (UHFto L-band) JS amplifier (MITEQ Corp.) with a specified noise figure of $\mathrm{NF}=0.6 \mathrm{~dB}$ and gain of $G=30 \mathrm{~dB}$.

Local on-chip detection of the small voltage signal via the coupling capacitance $\delta C_{\text {res }} \propto$ $\delta V_{\text {res }}$ is desirable, therefore an on-chip preamplifier is used (Fujitsu FHX35X) [22]. The transistor in this configuration serves as an impedance converter. An aerial view of the setup and the amplifier is depicted in figure 9. The magnetic field orientation was chosen to be parallel to the surface of the transistor. As seen the large input impedance has to be adjusted by placing in parallel a resistor of $10 \mathrm{M} \Omega$, a capacitor on the supply line of $47 \mathrm{nF}$ and an additional resistor of $1.5 \mathrm{k} \Omega$ on the output. Clearly, a limitation of this setup is given by the low gain and the still large input capacitance $C_{\text {in }} \gg C_{\text {res }}$ of the transistor.

The quantity of interest representing the sensitivity of mechanical cantilevers is typically given by the minimum detectable force. This force of rectangular cantilevers is limited by vibrational noise and is proportional to

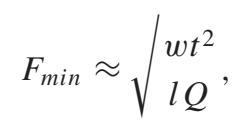

where $w, t$ and $l$ are the width, thickness and length of the beam. Similar equations are found for other types of micromechanical resonator. Obviously, the aim is to achieve a considerable size reduction of the structures leading to increased eigenfrequencies of the mechanical systems. The capacitive coupling between the gate electrode and the metallized resonator is estimated to be $\sim 200 \mathrm{aF}$ (see footnote 5), which translates, at an excitation power of $-42 \mathrm{dBm}$, into a force sensitivity of only $9.4 \times 10^{-14} \mathrm{~N}(\mathrm{~Hz})^{-1 / 2}$. Using this setup as an electrometer yields a charge sensitivity of $1.3 \times 10^{-3} \mathrm{e}(\mathrm{Hz})^{-1 / 2}$, which is three orders of magnitude better than previously measured [23]. Since the network analyser records amplitude and phase of the reflected signal it is also possible to implement a more sensitive lock-in technique for detection [24, 25].

\section{Sensor applications}

The ability to build mechanical devices on the nanometre scale allows us to reach a new regime for sensor applications. The already possible speed of operation, radiation hardness, the compatibility with standard silicon processing and the intrinsic sensitivity to their environment creates an increasing industrial demand [27].

The application which will be described in the following is focused on electrometry, since this scheme in principle is easily adopted to the detection of minimal quantities of mass, gas or magnetic materials. As will be seen, we achieve an extremely high sensitivity for charge detection. The setup is shown in figure 8: the resonator is probed by a network analyser and capacitive coupling of the side gates; i.e., the addition of charge $\delta q$ onto the electrode is analysed. From numerical evaluation a capacitive coupling between gate and beam in the 
R916

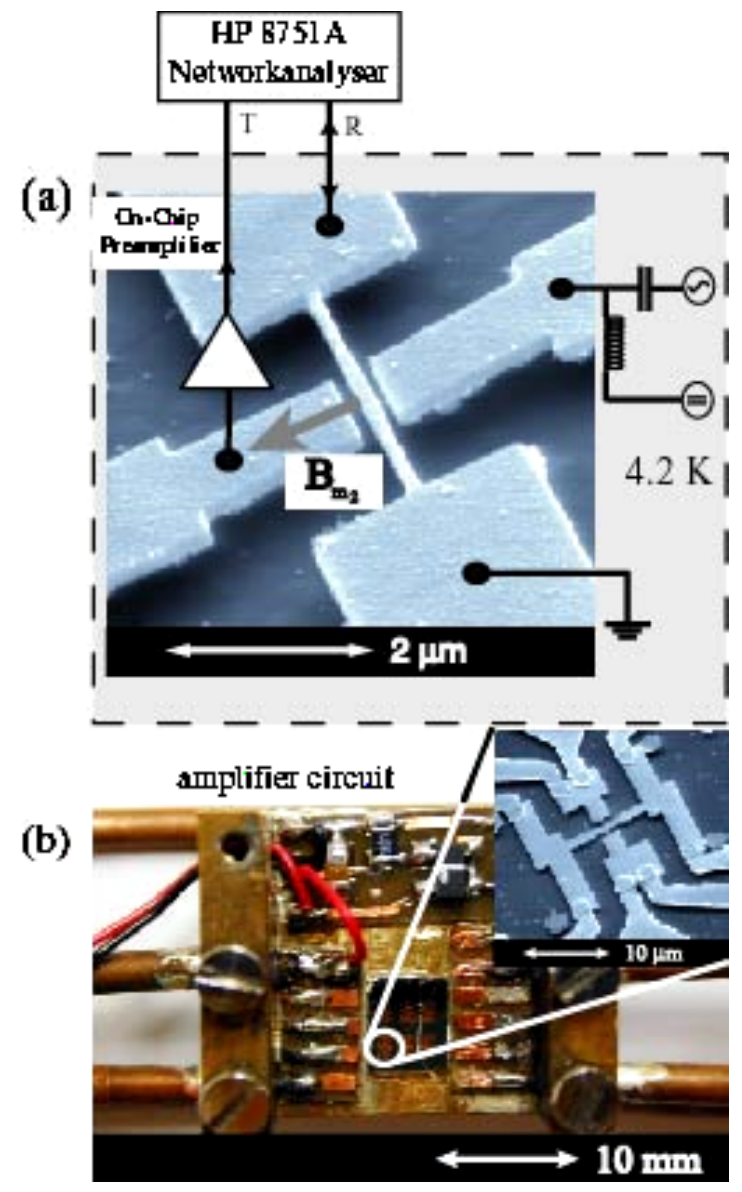

Figure 9. On-chip amplifier: one of the gates is coupled to the amplifier enabling capacitive detection of the beam's displacement. A magnified view of one of these is depicted in the inset.

linear regime of $C_{\mathrm{gb}} \cong 220 \mathrm{aF}$ is obtained. The frequency shift $\delta f$ of the mechanical resonance results from the capacitive coupling given by the electrostatic energy $E=q^{2} / 2 C$, where $q$ is the accumulated charge and $C$ the capacitance between resonator and gate. This term can be expanded with regard to the displacement amplitude $u=u(t)$ of the suspended beam, which yields for the electrostatic energy with $C=C(u)$ via a truncated Taylor expansion

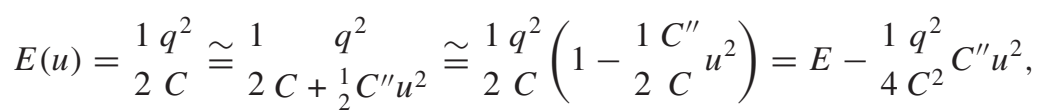

where $C^{\prime \prime}=\left.{ }^{\partial^{2} C(u)}{ }_{\partial u^{2}}\right|_{u=0}$ represents the second derivative of the capacitance with respect to the spatial coordinate $u$ at $u=0$. This gives with $q=C V$ a frequency shift of the mechanical resonance of the order of

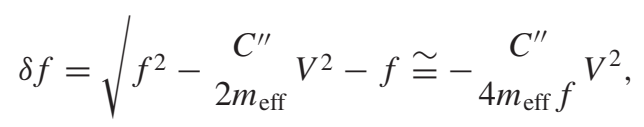

where $m_{\text {eff }}$ is the beam's effective mass (in our case $\sim 4.3 \times 10^{-16} \mathrm{~kg}$ ) and $V$ the applied gate voltage. In figure 10(a) the radio frequency (RF) response of the beam is depicted: applying 
(a)

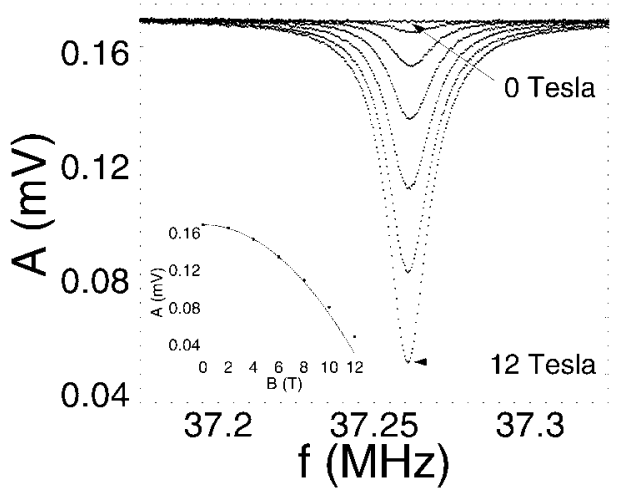

(b)

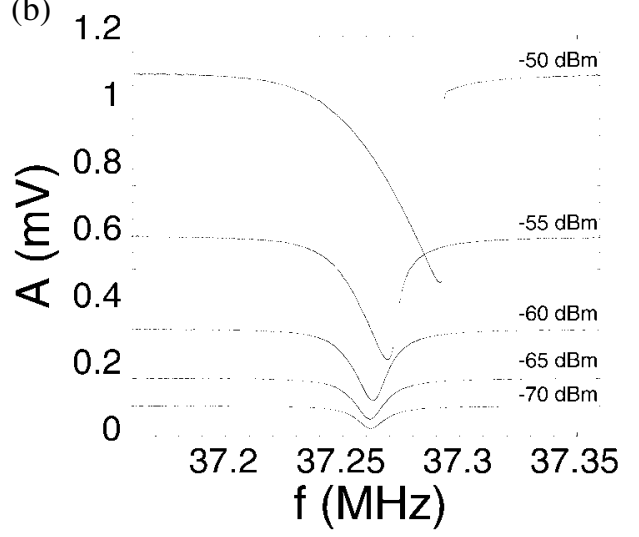

Figure 10. (a) Mechanical resonance at the $f=37.26 \mathrm{MHz}$ : excitation level is fixed at $-66 \mathrm{dBm}$ with peak amplitude increasing as $B^{2}$ (see inset). (b) Beam response driven into the nonlinear regime at $B=12 \mathrm{~T}$ with power levels increasing as indicated from -70 to $-50 \mathrm{dBm}$ [20].

a magnetic field in plane, an increase of the peak amplitude proportional to $B^{2}$ is found as plotted in the inset. The driving amplitude of the RF is $-66 \mathrm{dBm}$, ensuring linear response of the suspended beam. The FWHM of the resonance is $\delta f=(16 \pm 0.2) \mathrm{kHz}$, resulting in a mechanical quality factor at resonance of $Q=2330 \pm 30$. A large discrepancy compared to macroscopic mechanical oscillators with $Q \sim 10^{5}$ is obtained. This is explained by the coupling gas in the sample holder and the fact that the surface tension in these small devices naturally has a larger influence than in macroscopic systems.

In figure 10(b) the power coupled into the resonator is increased from -70 to $-50 \mathrm{dBm}$ where a strong nonlinear response is observed. In the present case the nonlinear response is identified by the distorted peak shape. Above a critical value of the excitation voltage the curve finally shows a bistability accompanied by a pronounced hysteresis. The transition occurs at about $-53 \mathrm{dBm}$, although an asymmetry of the peak structure is found at $-59 \mathrm{dBm}$. The nonlinearity is caused by the variation of the restoring force at the clamping points and can be modelled by adding a cubic term in the equation of motion of the beam [28]. Comparing our data with a model derived earlier in [29] excellent agreement is found (see section 2.1).

A closer inspection of the nonlinear response seen in figure 10 can be obtained with an external frequency source, while monitoring the absorption on the spectrum analyser. This allows approaching the hysteretic region around $f=37.287$ and $37.294 \mathrm{MHz}$ from larger and lower frequencies. In figure 11 such a measurement is shown: the inverted triangle $(\nabla)$ corresponds to an increasing frequency, while the triangle $(\triangle)$ represents the lowering branch. The applied power is $P_{\text {exc }}=-49 \mathrm{dBm}$ and the magnetic field $B=12 \mathrm{~T}$. Within this bistable region (width $\Delta f_{\text {hys }} \sim 7 \mathrm{kHz}$ ) the resonator is very sensitive to charge fluctuations on the nearby gate. Following this idea the suspended beam is a prime candidate to study stochastic resonance in a nanomechanical resonator at RF.

Optimum operating conditions are obtained by fixing the driving amplitude at the critical point with maximum slope as discussed in section 2.1 (traces in figure 12). The excitation power is set to $-52.8 \mathrm{dBm}$ and the magnetic field at $12 \mathrm{~T}$. As seen in the inset the peak position varies as the square of the gate voltage applied. The slope at the critical point $\mathrm{d} A /\left.\mathrm{d} f\right|_{f=f_{c}} \rightarrow \infty$ diverges, resulting in extremely sensitive amplification. It is important to note the enhancement of sensitivity with increasing gate voltage (see the inset of figure 12). The accuracy of the measurement can be further enhanced by determining the phase shift of the resonator. With 


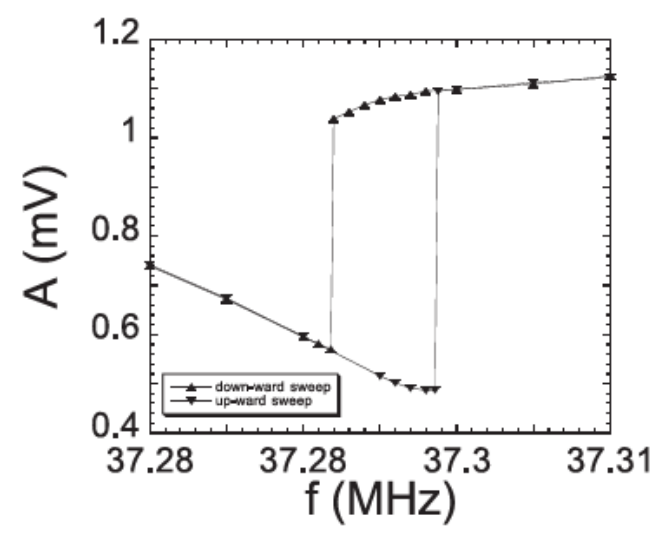

Figure 11. Hysteretic response of the excited beam [20]: the inverted triangle $(\nabla)$ indicates the signal with rising frequency, while the triangle $(\Delta)$ represents lowering of the frequency $\left(P_{\text {exc }}=-49 \mathrm{dBm}\right.$ and $\left.B=12 \mathrm{~T}\right)$.

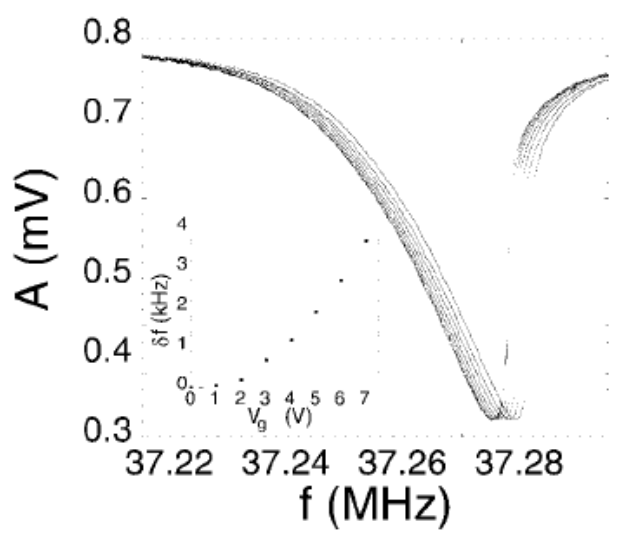

Figure 12. Operating the resonator in the transition region at $-52.8 \mathrm{dBm}$ with maximum signal sensitivity. Resonance traces are shifted by an applied gate voltage [20]. Note the shift of the critical point when the gate voltage is varied (inset).

such a setup it was possible to obtain a sensitivity of $\sim 1.0 \times 10^{-1} \mathrm{e}(\mathrm{Hz})^{-1 / 2}$. As before, the operating point is adjusted in the transition region at the critical point (as indicated in figure 12). Biasing the resonator to $V= \pm 4 \mathrm{~V}$, a charge resolution of $\delta q=n e=70 e$ is derived.

As seen nanomechanical resonators lend themselves to straightforward sensor applications at high frequencies, e.g. sensors using the internal strain of MBE-grown layers seem to be promising [30], also cantilevers made from polymer materials are coming into focus [31]. The measurement scheme discussed in this section for electrometry is easily altered to allow, e.g., mass sensing with high sensitivity. Apart from speed and resolution, the main advantage of the presented techniques is the compatibility regarding processing. Another application for NEMS is found in communication technology, where mechanical oscillators can be applied for signal transduction, filtering and mixing, as shown in the following section. 


\section{NEMS in the nonlinear regime}

The physics of nonlinear dynamics has been studied widely in macroscopic mechanical systems like the classical driven pendulum. With the advances in nanostructuring it is possible to build mechanical devices on the nanometre scale with eigenfrequencies of the order of several hundred MHz. Furthermore, the small dimensions offer the unique possibility to investigate nonlinear properties of a driven mechanical system approaching the quantum limit. In this section operation of NEMS in the nonlinear regime is demonstrated in order to investigate higher order mechanical mixing at RFs. These mixing properties are also of great importance for signal processing and exemplify nonlinear dynamics on the nanometre scale. The nonlinear response then is compared to $n$th order perturbation theory and nonperturbative numerical calculations.

The Duffing equation describes a deterministic system which can be brought into the chaotic regime by choosing a specific set of parameters. The system then is very sensitive to the boundary conditions and shows irregular orbits. In reality dissipation leads to a convergence of the chaotic trajectories onto attractors $A$ of dimension $n-1$, where $n$ is the phase space dimension. The hysteresis found for the nanomechanical resonators is a good example for an attractor, since several distinct points in phase space are stable conditions for the resonator.

Analysing chaotic motion is possible by tracing the Fourier transform $X(\omega)$ of a time evolution $X(t)$ :

$$
X(\omega)=\lim _{T \rightarrow \infty} \frac{1}{T} \int_{0}^{T} x(t) \mathrm{e}^{-\mathrm{i} \omega t} \mathrm{~d} t,
$$

which leads to the spectrum recorded by the spectrum/network analyser

$$
R(\omega)=|X(\omega)|^{2} .
$$

In the case of mechanical resonators excited at around 10-100 MHz sampling rates $>10 \mathrm{GHz}$ are required to record at least 100 data points, which is very difficult to achieve [24]. On the other hand a periodic oscillation is characterized by its line spectra, i.e. during the transition into chaos in these spectra subharmonics will emerge. Hard chaos is finally identified by a continuous background and number of discrete resonances.

Following Feigenbaum's analysis a driven nonlinear system shows a universal period doubling, which is characterized by the Feigenbaum constant $\delta=4.6692016$...; i.e., given that $F_{n}$ is the amplitude of the external excitation with a period $T_{n}=2^{n} T_{0}$, we find for the modulation parameter $F$

$$
\begin{gathered}
F_{n+1}-F_{n} \\
F_{n+2}-F_{n+1}
\end{gathered}=\delta .
$$

Writing the Duffing equation in the form of a double-well potential as shown in figure 13

$$
\ddot{x}+2 \mu \dot{x}-\beta x+k_{3} x^{3}=F \cos \omega t,
$$

with $\beta, k_{3}>0$; if $\beta>0$, this implies a positive back-action force. This can only be countered by a sufficiently large restoring force, given by the nonlinear term $k_{3} x^{3}(y)$. Following [7] the minimal amplitude of the external force $F$ for which chaos appears can be estimated,

$$
F>\begin{gathered}
4 \sqrt{ } 2 \mu \sqrt[3]{\beta} \\
3 \pi \omega \sqrt{ } k_{3}
\end{gathered} \cosh \left(\begin{array}{c}
\pi \omega \\
2 \sqrt{ } \beta
\end{array}\right) .
$$

For a certain choice of parameters $2 \mu=0.1, \beta=1, k_{3}=1$ and $\omega=1$, equation (38) reads

$$
\ddot{x}+0.1 \dot{x}-x+x^{3}=F \cos t .
$$




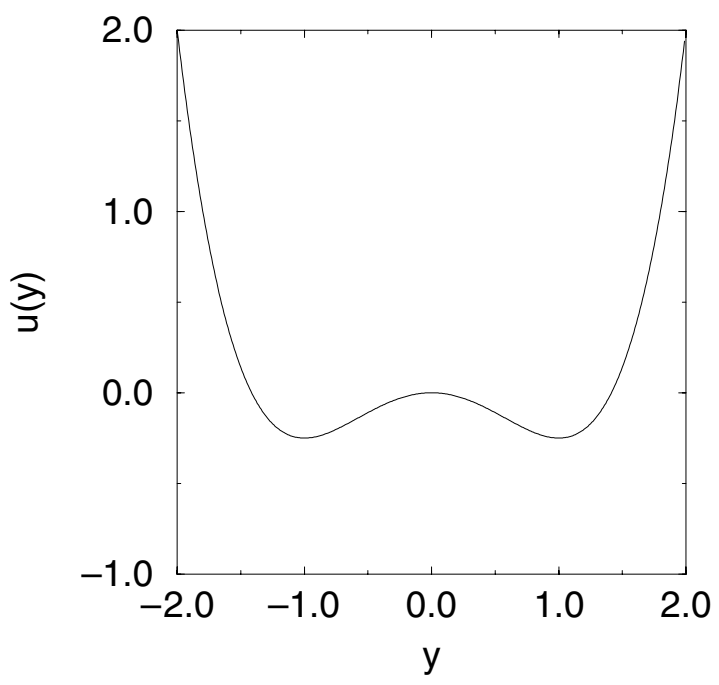

Figure 13. The double-well potential denoted as $u(y)=-\frac{y^{2}}{2}+\frac{y^{4}}{4}$.

The potential for these parameters is

$$
u(x)=-\frac{x^{2}}{2}+\frac{x^{4}}{4}
$$

as seen in figure 13. The excitation amplitude at which chaos should emerge is found to be $F>0.07$ (equation (39)). The differential equation (40) was solved and analysed according to [24]. The calculated results are shown in the phase space plot in figure 14. For small amplitudes $F=0.3$ the phase space plots in figure 14 are symmetrical with respect to both axes and the trajectory is periodic. In the right panel the oscillations of the system are shown after ten cycles. Upon increasing the amplitude to $F=0.4$ the plot is slightly asymmetric, this being the first indication of the path into chaos [32]. At $F=4.5$ period doubling occurs, clearly seen in the time evolution. Above $F=4.9$ the pattern is completely chaotic (see figure 15).

In order to estimate the parameter space where chaotic motion is expected the Duffing equation has to be written as

$$
\omega_{0}^{2} x^{\prime \prime}+2 \mu \omega_{0} x^{\prime}+\omega_{0}^{2} x+k_{3} x^{3}=F \cos \left(\begin{array}{c}
\omega \\
\omega_{0}
\end{array}\right),
$$

where $\tau=\omega_{0} t$ and $x^{\prime}=\partial x / \partial \tau$. Dividing by $\omega_{0}^{2}$ and substituting $x \mapsto y / \sqrt{k_{3} / \omega_{0}^{2}}$

$$
y^{\prime \prime}+2 D y^{\prime}+y+y^{3}=B \cos \left(\begin{array}{cc}
\omega & \\
\omega_{0} & \tau
\end{array}\right)
$$

with $D={ }_{\omega_{0}}^{\mu}$ and $B=\underset{\omega_{0}^{3}}{F} \sqrt{ } k_{3}$. A simplified form is given by

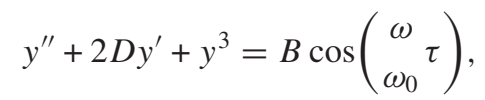

which is equation (43) without the linear term for the restoring force $\left(\mathcal{D F}[y]=y^{\prime \prime}+2 D y^{\prime}+y^{3}\right)$. From this equation a parameter plot can be obtained (see [24]), which allows us to determine approximate values for the appearance of chaos in a nanomechanical system. We estimate for 

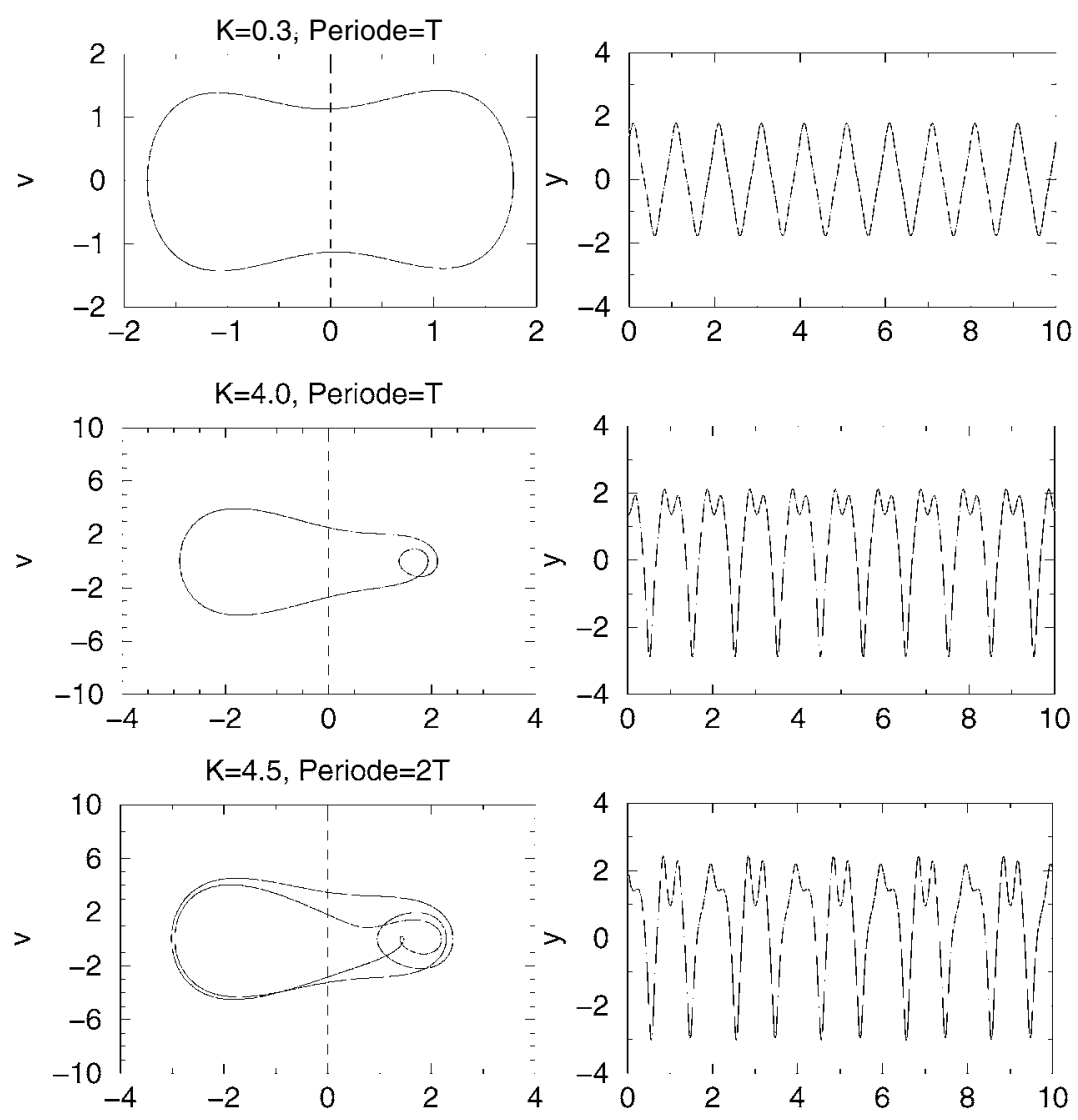

$\mathrm{K}=4.84$, Periode $=4 \mathrm{~T}$

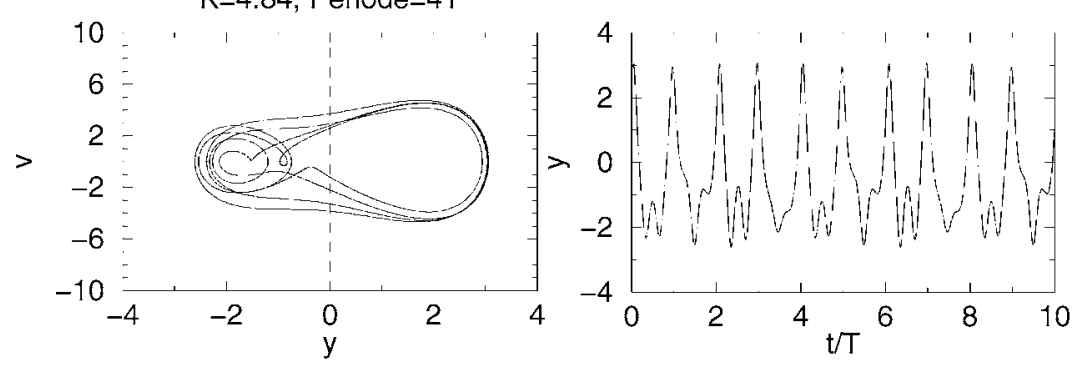

Figure 14. Numerically determined phase space diagrams (left) and time evolution (right) for equation (38) with varying amplitude $F \equiv K$.

fairly long resonators the transition into the chaotic regime to occur for $B>12 \mathrm{~T}$ under large excitation powers, but at low eigenfrequencies of $f_{0}<10 \mathrm{MHz}$.

A possible way to find the degree of nonlinearity and to trace the onset of deterministic chaos is by applying the mechanical resonator as a mixing element: in figure 16 the RF response of the beam near resonance is depicted for increasing magnetic field strength $B=0,1,2, \ldots, 12 \mathrm{~T}$. The excitation power of the spectrum analyser was fixed at $-50 \mathrm{dBm}$. The mechanical quality factor of the particular resonator under test in the linear regime is $Q=2330$. As seen the profile of the resonance curve changes from a symmetric shape at 


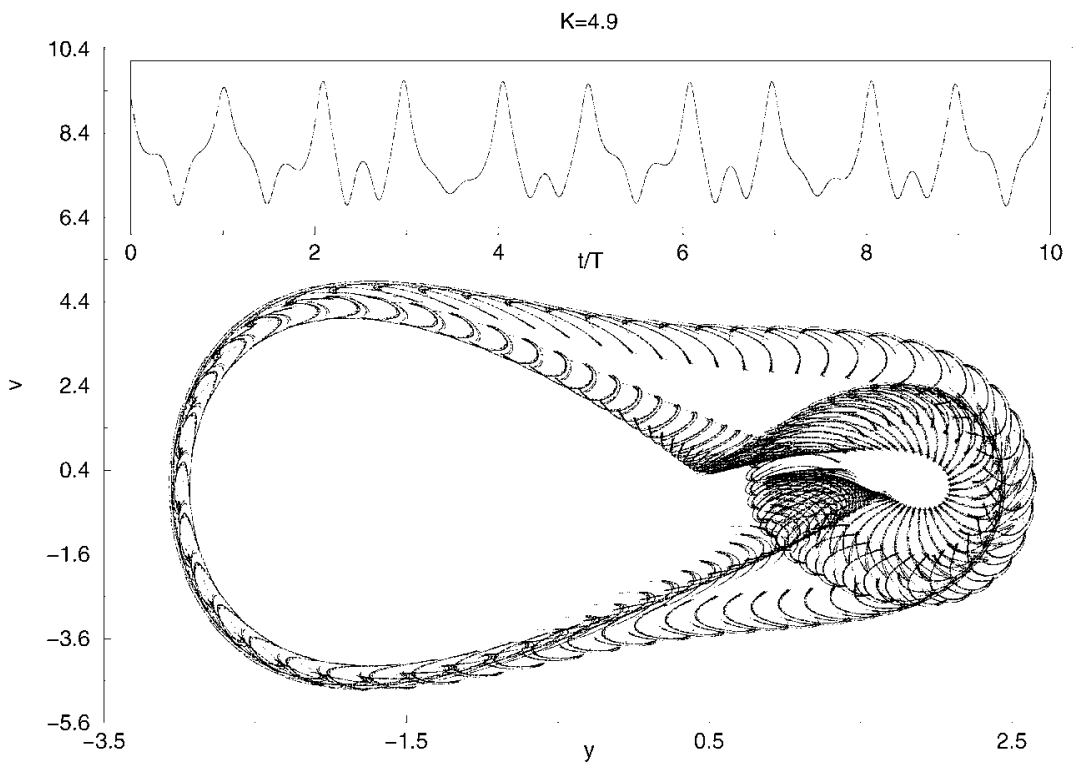

Figure 15. Phase space and time evolution for equation (38) at $F=4.9$.

moderate fields to an asymmetric, sawtooth shape at large field values, characteristic of an oscillator operated in the nonlinear regime. As already mentioned the nonlinearity can be described by the Duffing equation $\mathcal{D} \mathcal{F}[x(t)]=F(t)$ [32]. The external driving $F(t)$ is given by the Lorentz force

$$
F(t)={ }_{m_{\mathrm{eff}}}^{l B} I(t)={ }_{m_{\mathrm{eff}}}^{l B} I_{0} \cos (2 \pi f t),
$$

where $l=1.9 \times 10^{-6} \mathrm{~m}$ is the effective length and $m_{\text {eff }}=4.3 \times 10^{-16} \mathrm{~kg}$ is the effective mass of the resonator, $B$ is the magnetic field and $I_{0}$ the input current.

Solving the Duffing equation and computing the amplitude of the oscillation as a function of the driving frequency $f$ for several excitation strengths reproduces the measured curves shown in figure 16. The solutions at large power exhibit a region where three different amplitude values coexist at a single frequency. This behaviour leads to bistability in the measurements at high powers [20].

Turning now to the unique properties of the nonlinear nanomechanical system: by applying two separate frequency sources as sketched in figure $8(\operatorname{setup} \beta)$ it is possible to demonstrate mechanical mixing [33], as shown in figure 17. The two sources are tuned to $f_{1}=37.28 \mathrm{MHz}$ and $f_{2}=37.29 \mathrm{MHz}$ with constant offset and equal output power of $-48 \mathrm{dBm}$, well in the nonlinear regime. Without applying a magnetic field the two input signals are simply reflected. Above a critical field of $B \simeq 8 \mathrm{~T}$ higher order harmonics appear. Increasing the field strength, a multitude of satellite peaks evolves. As seen the limited bandwidth of this mechanical mixer allows effective signal filtering. Variation of the offset frequencies leads to data similar to those presented in figure 18: excitation at $-48 \mathrm{dBm}$ and $B=12 \mathrm{~T}$ with the base frequency fixed at $f_{1}$ and varying the sampling frequency in $\mathrm{kHz}$ steps from $f_{2}$ to $f_{1}$ yields satellites at the offset frequencies $f_{1,2} \pm n \Delta f, \Delta f=f_{1}-f_{2}$.

The nanomechanical system is modelled as a Duffing oscillator with a driving force

$$
F(t)=F_{1} \cos \left(2 \pi f_{1} t\right)+F_{2} \cos \left(2 \pi f_{2} t\right),
$$




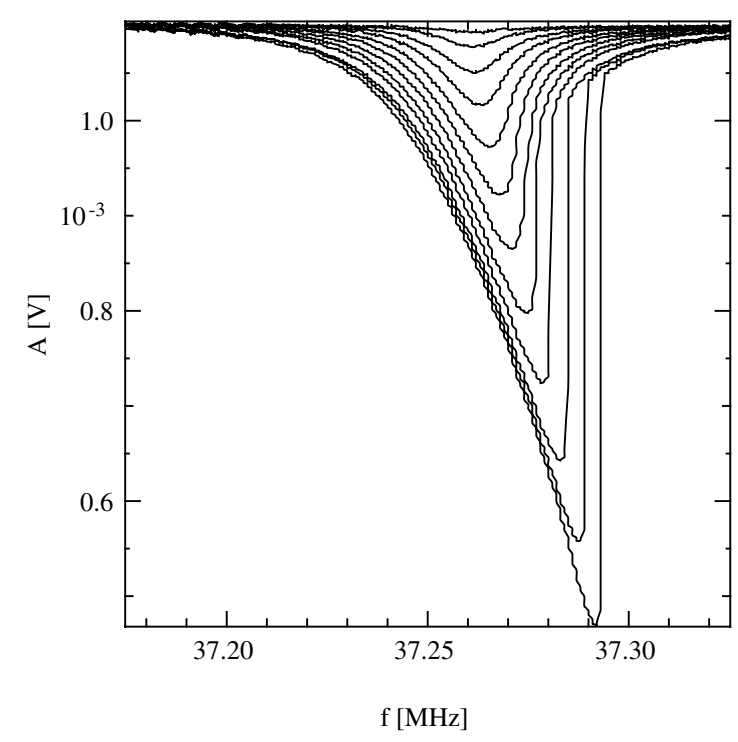

Figure 16. Characterization of the nonlinear response of the suspended beam: ramping the magnetic field from $0 \mathrm{~T}$ up to $12 \mathrm{~T}$ at large output power with constant driving amplitude around $-45 \mathrm{dBm}$ (cf figure 10).

with two different, but neighbouring, frequencies $f_{1}$ and $f_{2}$. Before presenting the numerical solution of the Duffing equation for the driving forces (equation (46)) an analysis is performed based on $n$ order perturbation theory [5] to explain the generation of higher harmonics. Expanding

$$
x=x_{0}+\eta x_{1}+\eta^{2} x_{2}+\cdots,
$$

where it is assumed that the (small) parameter $\eta$ is of the order of the nonlinearity $k_{3} x^{3}$, and inserting this expansion yields equations for the different orders in $\eta$. In zeroth order this leads to

$$
\ddot{x}_{0}+\mu \dot{x}_{0}+\omega_{0}^{2} x_{0}=F_{1} \cos \left(2 \pi f_{1} t\right)+F_{2} \cos \left(2 \pi f_{2} t\right),
$$

first order to $\ddot{x}_{1}+\mu \dot{x}_{1}+\omega_{0}^{2} x_{1}+k_{3} x_{0}^{3}=0$, and to similar equations for higher orders. After inserting the solution of equation (48) into the first order equation and assuming $f_{1} \approx f_{2} \approx f_{0}=\omega_{0} / 2 \pi$, two types of peak can be extracted: one peak is located at $3 f_{0}$. Peaks of the other type are found at frequencies $f_{i} \pm \Delta f$. Proceeding along the same lines in second order perturbation theory we obtain peaks at $5 f_{0}$ and $f_{i} \pm 2 \Delta f$. Accordingly, owing to the cubic nonlinear term, $n$th order peaks are generated at $(2 n+1) f_{0}$ and $f_{i} \pm n \Delta f$. While the $(2 n+1) f_{0}$-peaks could not be observed, the whole satellite family $f_{i} \pm n \Delta f$ is detected in the experimental power spectra (see figure 17). The perturbative approach yields the correct peak positions and, for $B<4 \mathrm{~T}$, also the peak amplitudes. However, in the hysteretic, strongly nonlinear regime a nonperturbative numerical calculation proves necessary to explain quantitatively the measured peak heights. The eigenfrequency is $f_{0}=37.26 \mathrm{MHz}$ as seen from figure 16 in the linear regime. The nonlinearity $k_{3}$ is estimated from the shift [5]

$$
\delta f(B)=f_{\max }(B)-f_{0}=\begin{gathered}
3 k_{3}\left[\Lambda_{0}(B)\right]^{2} \\
32 \pi^{2} f_{0}
\end{gathered}
$$

in frequency $f_{\max }$ at maximum amplitude in figure 16. In first order the displacement of the beam is given by $\Lambda_{0}(B)=l I_{0} B /\left(4 \pi f_{0} \mu m_{\text {eff }}\right)$. Relation (49) yields with $I_{0}=1.9 \times 10^{-5} \mathrm{~A}$ a value of $k_{3}=9.1 \times 10^{28}(\mathrm{~m} \mathrm{~s})^{-2}$. 

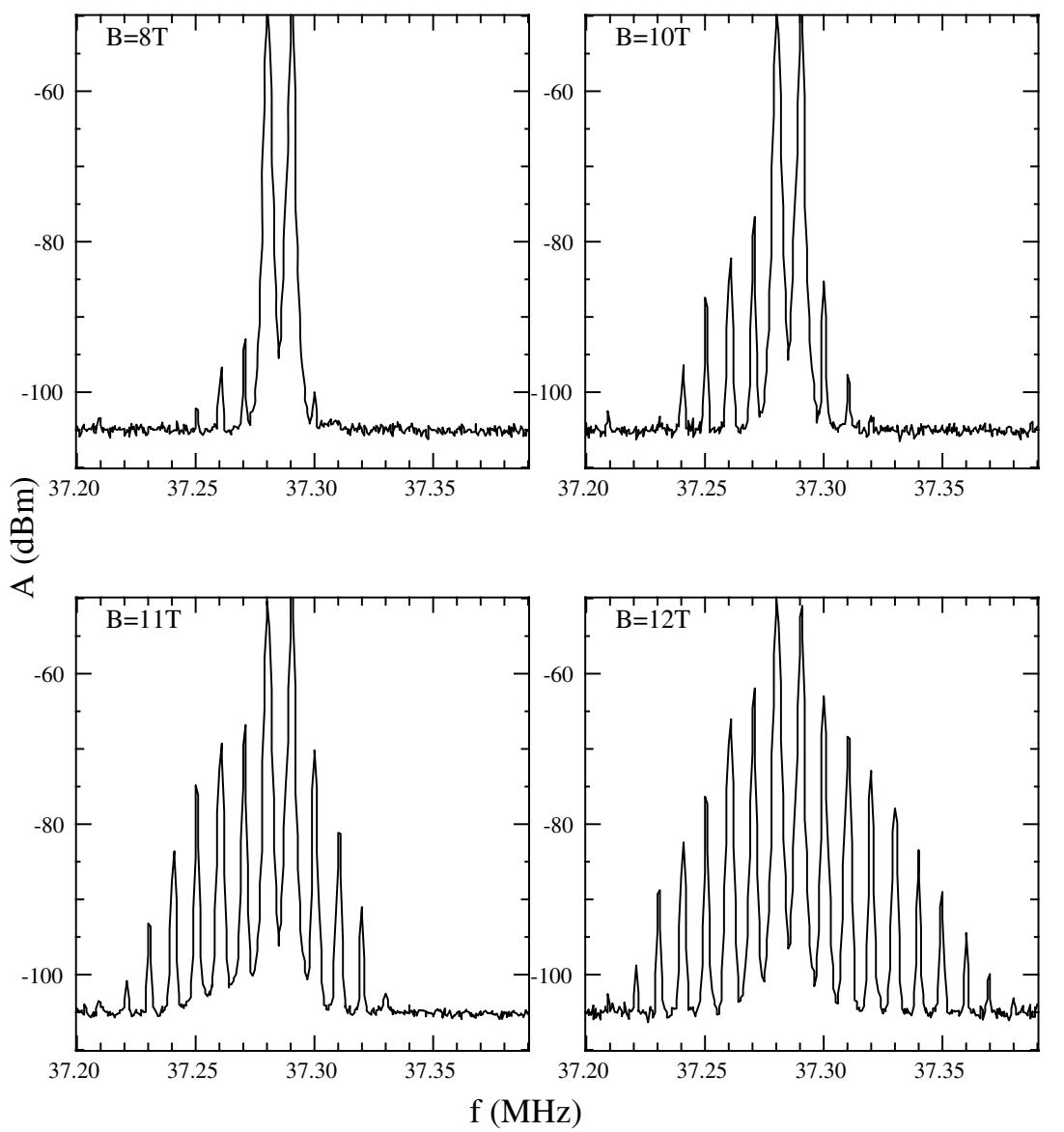

Figure 17. Two synthesizers (setup $\beta$ in figure 8) running at frequencies of $f_{1}=37.28 \mathrm{MHz}$ and $f_{2}=37.29 \mathrm{MHz}$ with constant offset (output power $-48 \mathrm{dBm}$ ) induce higher order harmonics as a result of mechanical mixing by the nanoresonator in the nonlinear regime $(B>8 \mathrm{~T})$.

First $x(t)$ is computed by numerical integration of the Duffing equation with a driving force as in equation (46) and $F_{1}=F_{2}=l B I_{0} / m_{\text {eff }}$. Then the power spectrum is calculated from the Fourier transform $X(\omega)$ of $X(t)$ for large times (beyond the transient regime). For a direct comparison with the measured power $P$ in figure 17 we employ $P \simeq R I_{\text {ap }}^{2}$. Here $R$ is the resistance of the electromechanical circuit and $I_{\text {ap }}=\left[4 \pi f_{0} \mu m_{\text {eff }} /(l B)\right] \hat{X}(\omega)$ the applied current, in close analogy to the relation between displacement $\Lambda_{0}$ and $I_{0}$.

The numerically obtained power spectra are displayed in figure 19: the emitted power for the same parameters as in figure 17 is shown. The positions of the measured satellite peaks, $f_{i} \pm n \Delta f$, and their amplitudes are in good agreement with the numerical simulations for the entire parameter range shown. Note that the height of the two central peaks in figure 17 cannot be reproduced by the simulations, since they are dominated by the reflected input signal.

The numerical results in figure 19 clearly show the evolution of an increasing number of peaks with growing magnetic field, i.e. increasing driving amplitude. As in the experiment, the spectra exhibit an asymmetry in number and height of the satellite peaks while switching from lower to higher frequencies by increasing the magnetic field from 8 to $12 \mathrm{~T}$. This behaviour can 


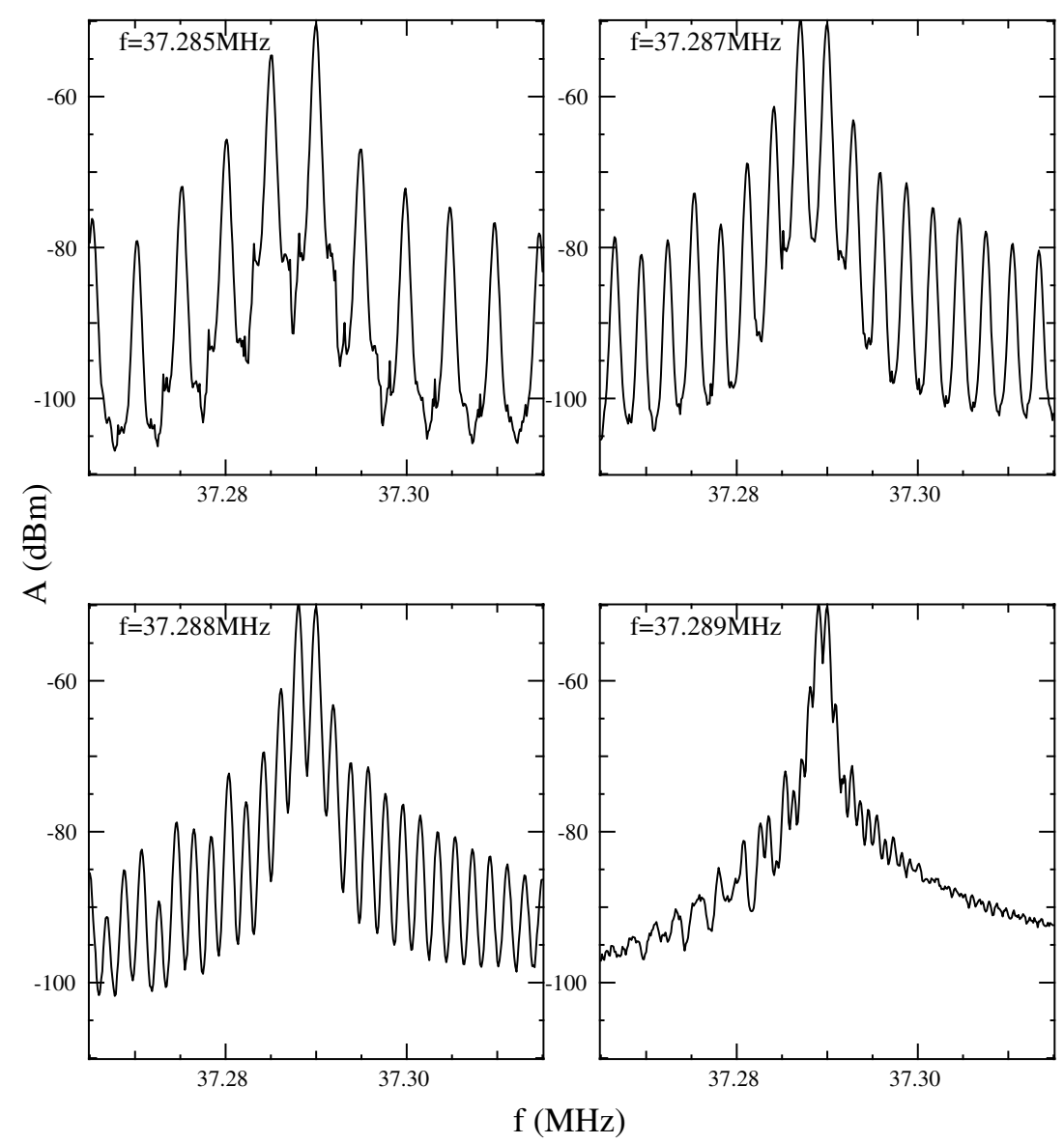

Figure 18. Two-source excitation at $-48 \mathrm{dBm}$ and $B=12 \mathrm{~T}$ : base frequency is $f_{1}=37.290 \mathrm{MHz}$, while the sampling frequency is varied in $1 \mathrm{kHz}$ steps from $f_{2}=37.285$ to $37.290 \mathrm{MHz}$ (as denoted in the graphs). As seen the spacing of the harmonics follows the offset frequency $\Delta f=f_{1}-f_{2}$.

be understood from equation (49) predicting a shift $\delta f$ in resonance frequency with increasing magnetic field. This shift is reflected in the crossover in figures 17 and 18 . For $B=8 \mathrm{~T}$ the amplitudes of the satellite peaks are larger on the left than on the right side of the two central peaks. As the field is increased the frequency shift drives the right-hand side satellites into resonance, increasing their heights.

The power spectra in figures 17 and 18 are rather insensitive to changes in magnetic field for $B<8 \mathrm{~T}$ compared to the rapid evolution of the satellite pattern for $8 \mathrm{~T}<B<12 \mathrm{~T}$. The analysis shows that this regime corresponds to scanning through the hysteretic part in the amplitude/frequency (or amplitude/ $B$-field) diagram, involving abrupt changes in the amplitudes. The resonator studied is strongly nonlinear but not governed by chaotic dynamics. Similar setups should allow for entering the truly chaotic regime. In further studies on the nanobeams in the nonlinear regime mechanical mixing was used as a probing mechanism. Upon reducing the frequency offset $\delta f$ between the two sources, the envelope function of the mixing product shows a clear periodicity as marked in figure 18. In other measurements a first bifurcation and thus a hint for the cross over of the system from simple nonlinear response into the chaotic regime was found [9]. 

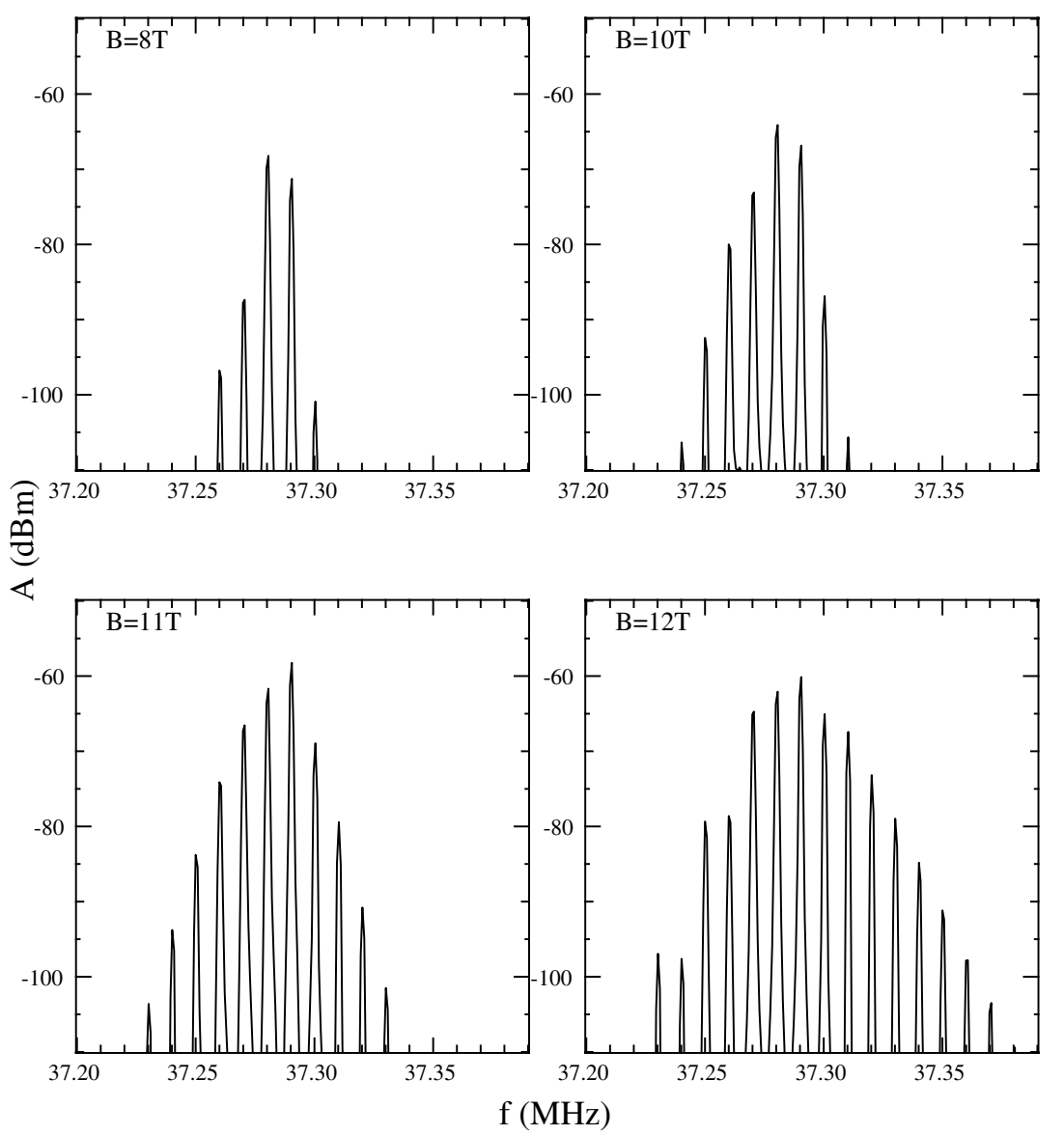

Figure 19. Calculation of the power spectra from the numerical solution of the Duffing equation assuming an external force equation (46) for the same driving frequencies as used in figure 17. Variation of magnetic field $B=8,10,11$ and $12 \mathrm{~T}$.

A requirement for entering the chaotic regime is the detection of harmonics of the mechanical base frequency. The resonance frequencies are calculated straightforwardly by FEM; we only recently observed harmonics and sub-harmonics. In figure 20 a first measurement on the detection of a harmonic is presented. Using harmonics will allow us to further increase the force sensitivity, by pumping the nanomechanical system on the fundamental mode while probing on one of the harmonic modes.

These studies open up a wide range of applications, especially for signal processing and scanning probe techniques [34]. The experimental results are in very good agreement with numerical calculations based on a generalized Duffing equation, a prototype of a nonlinear oscillator. Hence these mechanical resonators allow for studying nonlinear, possibly chaotic dynamics on the nanometre scale.

\section{Parametric amplifier}

In the above sections the advantages of NEMS have been outlined. One of the major advantages as compared to MEMS are the high frequencies of operation already achieved. Naturally, this 


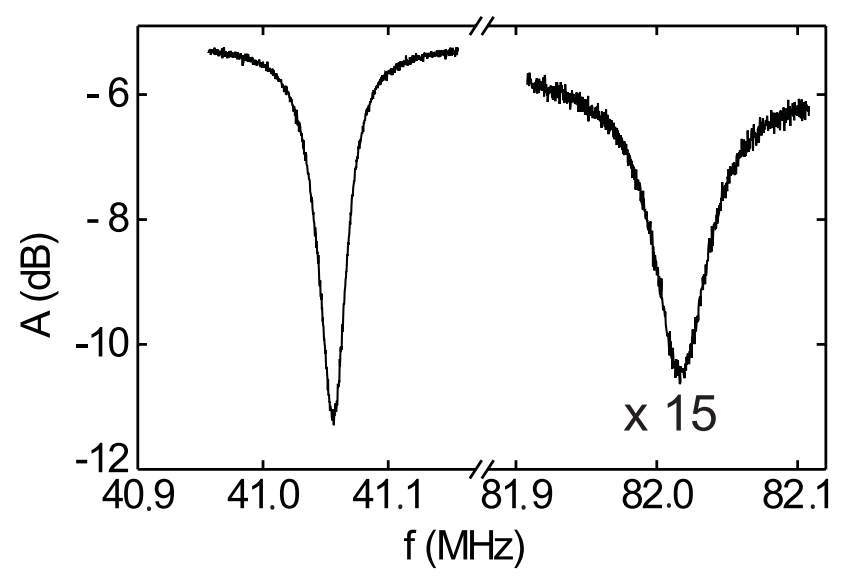

Figure 20. RF spectrum of the reflected power for a resonator: shown is the base frequency $f_{0}=41.05 \mathrm{MHz}$ and the first harmonic at $2 \times f_{0}$.

makes NEMS a favourite device for future integration of on-chip signal processing. However, the fundamental drawback of NEMS is their small size, since this inherently limits the signal strength with which, e.g., displacement of a resonant cantilever can be detected. Consider the standard example of a doubly clamped beam, excited by magneto-motive driving. The induced electromagnetic fields caused by the moving wire are then recorded. The signal strength depends on several parameters, among these the displacement of the suspended beam. The shorter the beam the smaller the induced voltage, hence a resonator with a length less than $400 \mathrm{~nm}$ will induce only a marginal signal.

A possibility to overcome this severe limitation is given at hand by using parametric resonance. Generally, one finds that the condition for parametric resonance at frequency $f$ is given by $f=2 f_{0} / n$, where $n$ is an integer [3]. The same principle holds for a huge variety of different mechanical as well as electronic systems. Naturally, it is also found for MEMS, such as microscopic cantilevers [28, 36] and micro-actuators [37], which are valuable for scanning probe applications. Recently parametric amplification was demonstrated in micromechanical resonators at eigenfrequencies of some kilohertz [38, 39], but not in the RF range. The obvious approach to achieve RF operation is to scale down the already known sensors and actuators from MEMS to the nanometre scale. This increases the possible mechanical frequencies by orders of magnitude, allowing us to reach resonance frequencies in the gigahertz range.

In a straightforward scheme, the bandwidth limitation can easily be overcome by means of parametric frequency tuning of a phase-locked nano-electromechanical resonator, as shown in figure 21 [40]. Important for achieving the large increase in bandwidth is the effective phase coupling between the nanomechanical resonator and the electrical circuit driving the device. In the following the response of the suspended bridges in two different geometries is investigated. This ensures proper treatment of the nonlinear restoring forces caused by the clamping points.

Sample A has a length of $l=1.82 \mu \mathrm{m}$, a width of $w=200 \mathrm{~nm}$ and a height of $h=250 \mathrm{~nm}$, is clamped on both ends, and the magnetic field is oriented perpendicular to the sample plane. The other resonator (termed B) has a slightly different geometry: $l=4.8 \mu \mathrm{m}$, $w=170 \mathrm{~nm}$ and $h=240 \mathrm{~nm}$, while the magnetic field is fixed in plane, leading to an out-ofplane displacement. The two nanoresonators allow us to verify parametric frequency tuning for two different magnetic field configurations, by pumping them with a synthesizer at a frequency $f_{p} \sim f_{0}$ on one of the side gates. This mode couples capacitively to the mechanical resonator with an estimated capacitance of $C_{\text {gate }} \sim 0.2 \mathrm{fF}$ for sample A and $C_{\text {gate }} \sim 0.6 \mathrm{fF}$ for sample B. 


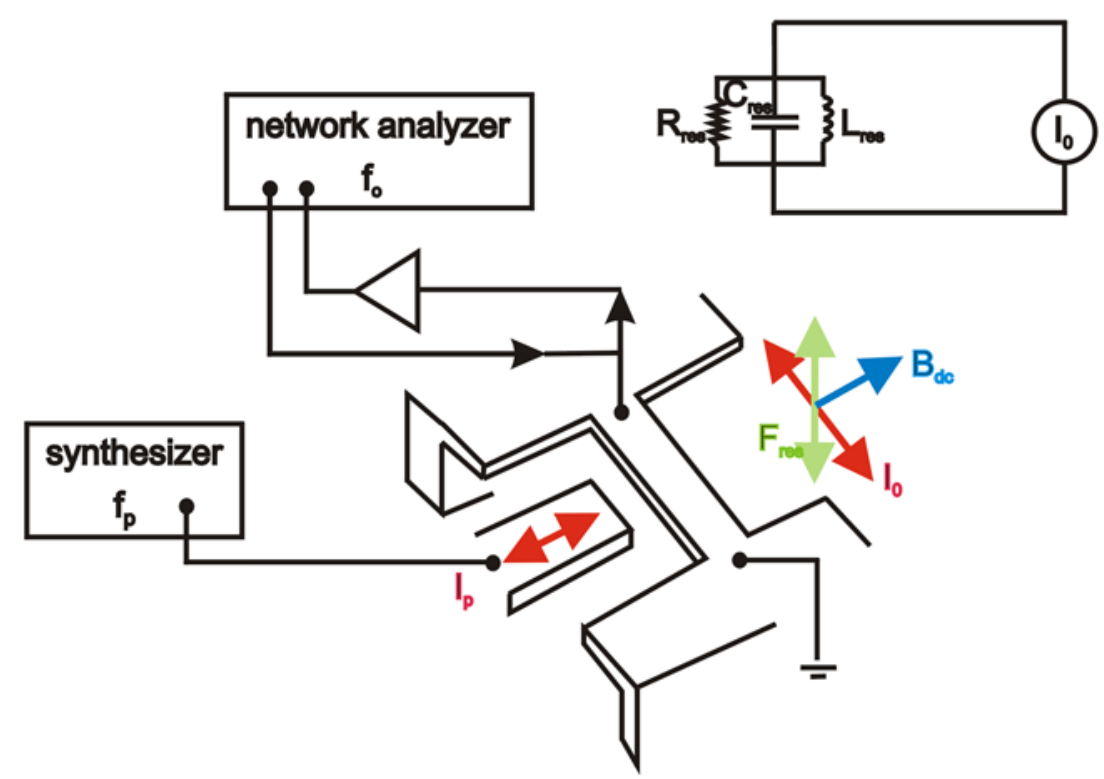

Figure 21. Experimental setup for parametric frequency tuning: adding the pumping frequency $f_{p}$ with a resulting current flow $I_{p}$ on one of the gate electrodes and determining the variation of the reflected power. Sketch of the sample configurations with the two different modes investigated: in both cases an alternating current $I_{0}$ is sent through the suspended bridge with the static magnetic field $B$ either in the sample plane or directed out of it, probing the strain dependence of the clamping points. The resulting Lorentz force leads to in-plane mechanical oscillations for sample A, and out-of-plane oscillations for sample B. The inset shows a circuit diagram with $R_{\text {res }}, C_{\text {res }}$ and $L_{\text {res }}$ used to model mechanical resonators.

In figure 22 (sample A) and figure 23 (sample B) the RF response is depicted for different pumping frequencies at a fixed magnetic field strength. The excitation power of the network analyser was fixed at $-50 \mathrm{dBm}$. The mechanical quality factor of the resonators under test in the linear regime is $Q^{\mathrm{A}}=1.37 \times 10^{3}$ and $Q^{\mathrm{B}}=2.73 \times 10^{3}$. The fundamental resonance frequency is given by $f_{0}=\omega_{0} / 2 \pi \cong(E / \rho)^{1 / 2} d / l^{2}$ and $d$ is the beam's thickness-for Au $E=8.0 \times 10^{10} \mathrm{~N} \mathrm{~m}^{-2}$ and $\rho=19.32 \times 10^{3} \mathrm{~kg} \mathrm{~m}^{-3}$ and for Si $E_{(100)}=1.7 \times 10^{11} \mathrm{~N} \mathrm{~m}^{-2}$ and $\rho=2330 \mathrm{~kg} \mathrm{~m}^{-3}$. The effective masses of the resonators are computed to be $m_{\text {eff }}^{\mathrm{A}}=6.15 \times 10^{-16} \mathrm{~kg}$ and $m_{\mathrm{eff}}^{\mathrm{B}}=9.67 \times 10^{-16} \mathrm{~kg}$. The theoretically estimated resonance frequencies are of the order of $f_{0}^{\mathrm{A}}=74 \mathrm{MHz}$ and $f_{0}^{\mathrm{B}}=72 \mathrm{MHz}$, while the eigenfrequencies obtained by Solvia are 95.93 and $81.7 \mathrm{MHz}$, respectively.

As seen in figure 22 the profile of most of the resonance curves follows a Lorentzian shape corresponding to the linear response regime. For the other resonator discussed below a transition from a symmetric resonance shape to an asymmetric, sawtooth shape is found, characteristic of an oscillator operated in the nonlinear regime (figure 23). As seen the resonance maximum shifts by $\delta f_{a c}=130 \mathrm{kHz}$ or vanishes completely for $f_{p}=f_{0}=$ 95.90 MHz (figure 22). A more complete view is also given in figure 22: the resonance structure is shown in grey scale representation, where dark blue regions correspond to energy absorption by the beam, i.e. a mechanical resonance, while the bright regions indicate zero displacement. The plot is spanned by the frequencies $f$ and $f_{p}$. Direct cross-talk is seen in the intersectioning line with linear slope. The mechanical resonance is found around $95.9 \mathrm{MHz}$ and is strongly distorted when the pumping frequency approaches $95.7 \mathrm{MHz}$. The mechanical oscillator then locks to $f_{p}=f_{0}=95.77 \mathrm{MHz}$, where the shift of the eigenfrequency is of the 

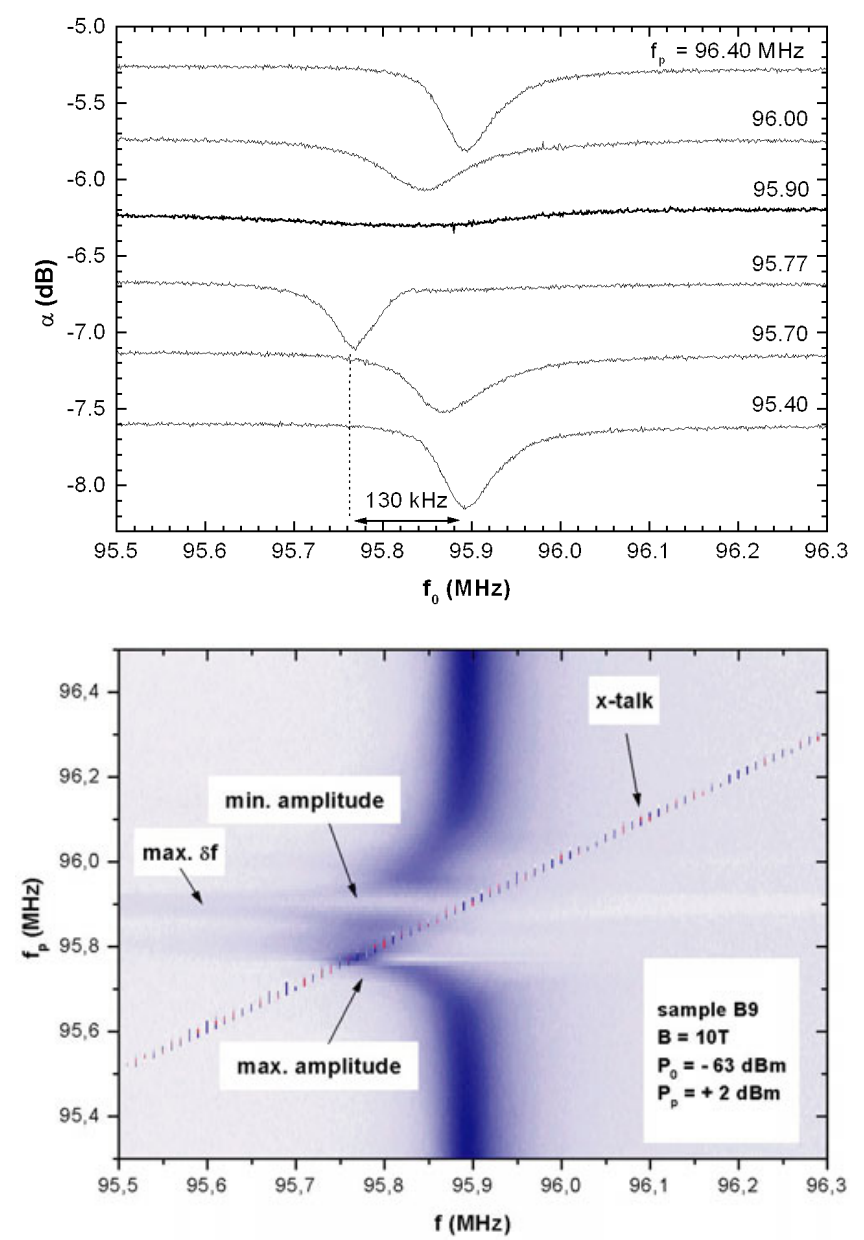

Figure 22. Top: typical response of a nanomechanical resonator around $95 \mathrm{MHz}$ (sample A) with different pumping frequencies $f_{p}$ applied as noted. The ac frequency leads to a shift of the resonance frequency of $\delta f_{a c}=130 \mathrm{kHz}$, compared to $\delta f_{d c}=4 \mathrm{kHz}$ for a dc bias on the gate electrode [20]. The data is taken at a magnetic field strength of $B=10 \mathrm{~T}$ and at $4.2 \mathrm{~K}$ with the power levels of the driving frequency at $P_{0}=-63 \mathrm{dBm}$ and of the pumping frequency at $P_{p}=2 \mathrm{dBm}$. Bottom: complete spectrum from the above shown data in a grey scale plot (resonance is depicted as a dark region).

order of $130 \mathrm{kHz}$. The power level of the driving signal at frequency $f$ is found to be relatively low at $P=-63 \mathrm{dBm}$, while a large field strength is required for the pumping signal at $f_{p}$ with $P_{p}=+2 \mathrm{dBm}$.

In conventional measurements a frequency shift corresponding to an effective bandwidth of $B \propto \delta f_{d c} \cong \pm 2 \mathrm{kHz}$ under $V_{d c}= \pm 3 \mathrm{~V}$ is found [20]. The large frequency shift of $\delta f_{a c} \cong \pm 130 \mathrm{kHz}$ is only possible since the mechanical properties of the beam are modified under the action of the RF signal in resonance with the mechanical circuit. A further increase of $f_{p}$ leads to a complete localization of the levers, marked by minimum amplitude. In between these two, the frequency shift $\delta f$ has its maximum albeit with a smaller amplitude. This corresponds to a softening of the mechanical resonance mode, i.e. the restoring force is changed under the action of a dynamic excitation. Such a behaviour is characteristic for frequency 
tunable parametric resonators. The parametric excitation obviously allows increasing the bandwidth by two orders of magnitude. An analogous effect is well known from experiments on optically active materials [41].

It was possible to realize an enhancement of parametric frequency tuning by using the slightly larger resonator (sample B). The resonator itself is excited well into the nonlinear regime as indicated by the resonance shape (see figure 23). A shift in eigenfrequency with opposite sign compared to sample A is measured. The bandwidth in this case is approaching $\delta f \sim 400 \mathrm{kHz}$ and is even larger compared to sample A, while the applied field strengths are of the same order of magnitude. Clearly, this larger signal intensity is due to the increase in length, while the sign changes result from the different interplay of the elastic moduli of $\mathrm{Au}$ and $\mathrm{Si}$ and hence the constants when the bridge is moving perpendicular to the sample plane. The increased noise in the tuning regime results from the direct cross-talk of the pumping frequency and can be eliminated by proper filtering of the response.

The force $F(t)$ acting on the nanoresonator due to the RF current in the capacitor is given by $F=q E$, where $E=E_{0} \mathrm{e}^{\mathrm{i} \omega_{p} t}$ is the electric pumping field and $q=\int I \mathrm{~d} t$, with $I=I_{0} \exp (\mathrm{i}(\omega t+\phi))$ the current running through the bridge. The phase $\phi$ is a free parameter of the system and the frequency is defined as usual as $\omega=2 \pi f$. One obtains $F \propto \exp \left(\mathrm{i}\left(\left(\omega+\omega_{p}\right) t+\phi\right)\right)$ for the experimental situation $\omega_{0} \approx \omega \approx \omega_{p}$, hence the signal at the capacitor is roughly twice the eigenfrequency $\omega_{0}$ of the nanoresonator, which leads to parametric resonance depending on the value of $\phi$. In the experimental situation the pumping signal $\omega_{p}=2 \pi f_{p}$ is ramped, while the other parameters are kept constant. As noted before it is found that when $\omega_{p}$ is approaching $\omega$ the two oscillations are synchronized, i.e. $\phi=$ constant. Coupling energy demands the condition $\phi=0$, which implies that the nanoresonator is functioning as a parametric resonator.

A very important difference between these two samples is found with respect to the nonlinear term, since for sample A not only the first nonlinear term $k_{3}$ has to be considered as for sample $\mathrm{B}$, but also a second term $k_{5}$. The effect of the nonlinearity on the parametric resonance can be summarized as follows: if the condition $\mu=k_{3}=k_{5}=F(t)=0$ is chosen, the Mathieu equation is obtained, which gives rise to an explosive growth of $x\left(\omega+\omega_{p}\right)$ in the region of parametric resonance. Introducing attenuation reduces the region of parametric resonance in parameter space. The nonlinearity, however, suppresses the strong growth of $x$, since it produces a frequency shift $\delta_{1}$ that finally leads to a detuning.

For nanoresonator B the parameter $k_{3}$ is constant for a large range of amplitude values, hence it is a 'tame' nonlinearity, and the assumption $k_{5}=0$ holds. For a constant input at the resonator the nonlinearity implies a constant frequency shift $\delta_{1}$. In this case the new condition for parametric resonance is expected to be $\omega+\omega_{p}=2\left(\omega_{0}+\delta_{1}\right)$, and not $\omega+\omega_{p}=2 \omega_{0}$ as in the linear case. It is worthwhile to note again that parametric resonance in this case does not drive $x$ into the region of explosive growth due to the detuning. Once the condition $\left(\omega+\omega_{p}\right)=2\left(\omega_{0}+\delta\right)$ is met, the amplitude of the displacement $x$ grows and detunes the system, decreasing the amplitude in turn and $\delta$. In this way the resonator remains always in balance around the new parametric resonance condition. However, a large amplitude is expected for the condition $\omega=\omega_{p}+\delta_{2}$. This new symmetry at $\omega=\omega_{p}+\delta_{2}$ is observed in figure 23 . It shows the amplitude absorption for sample $\mathrm{B}$ in a colour scale plot spanned by $f \sim \omega$ versus $f_{p} \sim \omega_{p}$. This figure is interpreted in the following way: when the nanoresonator is not synchronized, no parametric, but a nonlinear resonance is found. When the system is synchronized parametric resonance evolves with the resonance condition given by $\omega=\omega_{p}+\delta_{2}$.

Nanoresonator A, on the other hand, possesses a strong nonlinearity, since $k_{3}$ is not constant for large amplitudes. Furthermore, $k_{3}$ has a negative value which implies that for large amplitudes (taking $k_{3}<0$ and $k_{5}=0$ ) the displacement $x$ diverges. Obviously, the resonator 

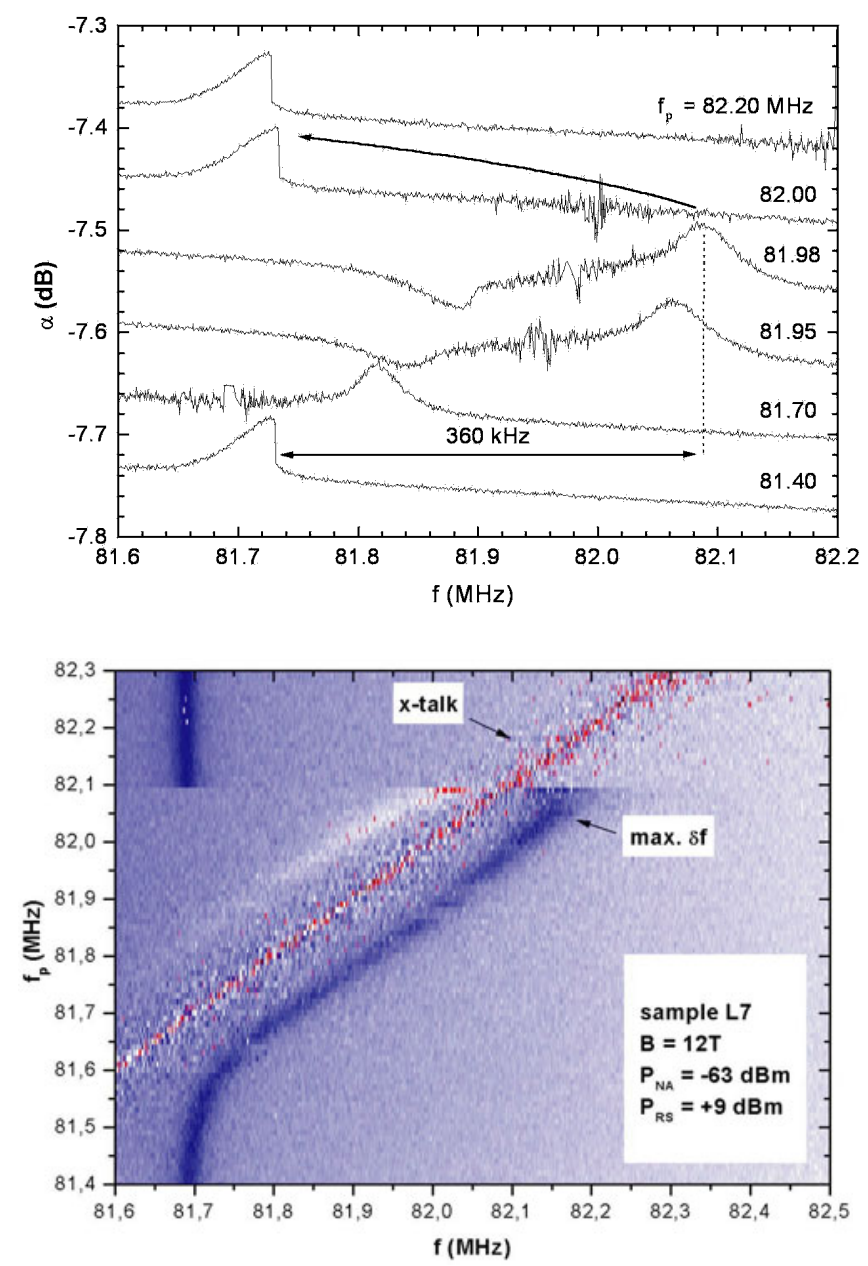

Figure 23. Line plots (top) and grey scale plots (bottom) of parametric resonance tuning of sample B. The resonator is operated in the nonlinear regime, indicated by the resonance shape. In contrast to sample $\mathrm{A}$ the frequency shift $\delta f_{a c}$ is positive and even larger, due to the different mechanical properties of this beam.

does not collapse and $x$ does not diverge, i.e. saturation effects caused by higher nonlinearities are present (modelled by the additional term $k_{5}$ ). The presence of two nonlinearities leads to a more complex picture for A compared to B. In this case it is expected that, when the system is not synchronized only a trivial nonlinear resonance at $\omega+\delta_{1}$ is observed. When the two frequencies synchronize parametric resonance kicks in and a complex scenario is built up by the interplay of the two nonlinear terms $k_{3}$ and $k_{5}$.

The achieved increase in bandwidth by two orders of magnitude will finally help in overcoming the most severe limitation of NEMS. A very promising experiment will be the combination of parametric frequency tuning and quantum noise squeezing for quantum nondemolition (QND) measurements in nanomechanical systems. 
Table 1. Resonance frequencies of a torsional resonator and corresponding $Q$-values.

$\begin{array}{cc}\text { Frequency }(\mathrm{MHz}) & \text { Quality factor } \\ 0.566 & 560 \\ 0.584 & 580 \\ 0.741 & 2470 \\ 1.27 & 1270 \\ 2.33 & 1165 \\ 3.66 & 1220 \\ 51.5 & 1198 \\ 54.08 & 3605 \\ 76.2 & 1520 \\ 78.4 & 3140 \\ 109.38 & 2956 \\ 237.5 & 2160 \\ 239.3 & 1710 \\ 318.1 & 1770\end{array}$

\section{Dissipation}

The crucial quantity of nanomachined resonators is the mechanical quality factor $Q$, since it is dominated by the energy dissipated in the device $[43,45]$. Dissipation in these structures is determined by external thermal phonons, i.e. radiation into clamping points, internal thermal phonons (other resonator modes), electrons in overlayers, surrounding gas, bulk defects (e.g. two-level systems), surface defects and adsorbates [42]. Nanomechanical resonators show in comparison to macroscopic ones $Q$-values of the order of only $10^{3}-10^{4}$ [44], where the definition of $Q$ is

$$
Q=\begin{gathered}
\text { energy in each cycle } \epsilon \\
\text { dissipated energy in each cycle } \epsilon_{\text {diss }}
\end{gathered} .
$$

Considering magneto-motively driven resonators

$$
E={ }_{2}^{1} m_{\mathrm{eff}} \omega_{0}^{2} y_{0, \max }^{2}=\begin{gathered}
m_{\mathrm{eff}} K^{2} \\
8 \mu^{2}
\end{gathered}=\begin{aligned}
& L^{2} B^{2} I_{0}^{2} \\
& 8 m_{\mathrm{eff}} \mu^{2}
\end{aligned}
$$

The attenuation constant $\mu$ is connected to the $Q$-value via $\mu=\omega_{0} / 2 Q$. The total energy dissipated per cycle $\epsilon_{\text {diss }}$ is given as a sum of the different mechanisms, which will be discussed in the following. In table 1 the eigenmodes and the corresponding $Q$-values of a typical resonator are given. The large number of modes found is due to the more complex geometry compared to conventional beams. Interestingly resonances up to $0.4 \mathrm{GHz}$ were found-the $Q$-values on the other hand were all of the same order of magnitude. All resonances are indeed of mechanical origin and were taken in the linear response regime.

\subsection{Ohmic losses}

Exciting the resonator electromagnetically results in ohmic losses in the metallic top layer. The dissipated power in an ohmic resistor is defined as $P_{\text {diss }}=R I_{0}^{2}$, with a resistance $R$ of the metal and a driving current $I_{0}$. The resistance of the samples is usually found to be around $10^{-2}-10^{-1} \Omega$. The experimentally found value is of the order of $Q_{\max } \sim 1-5 \times 10^{4}$. Usage of superconducting metal will help to reduce this source of dissipation. In order to address the physics of dissipation in the ultimate limit of single electrons interacting with phonon modes of their semiconductor host crystal, the most promising approach is to machine freely suspended 

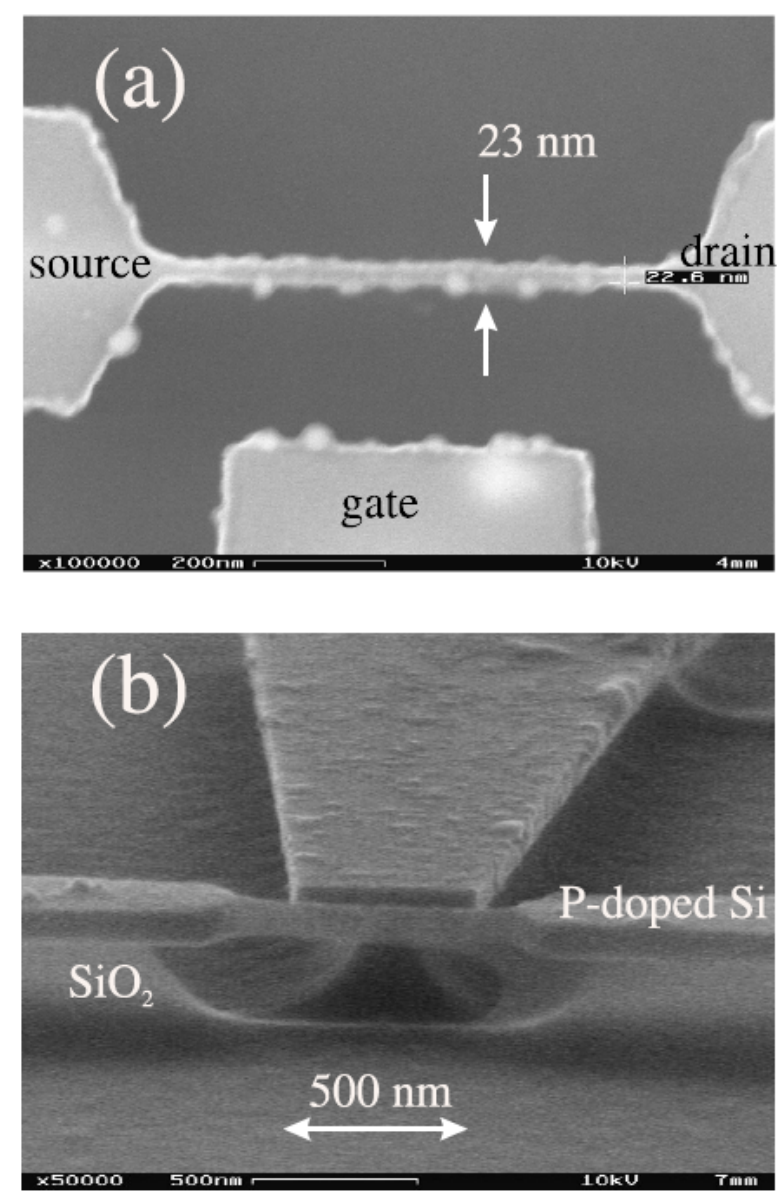

Figure 24. (a) Top view and (b) side view of the smallest suspended silicon nanowire fabricated with a width of $23 \mathrm{~nm}$. The highly doped wire is clearly underetched; the gate allows tuning of the carrier density in the wire.

nanobridges from doped semiconductor materials as shown in figure 24 . These bridges allow us then to study the relaxation between electrons and phonons $[46,47]$ in detail by tailoring the phonon mode spectrum.

Efforts were undertaken in nanostructuring of Si-based materials: highly p-doped suspended silicon wires were investigated at room temperature by Fujii $e t$ al [48]. Recently a beautiful experiment by Schwab et al [49] revealed that in suspended Si nanostructures the thermal conductance carried by phonons is quantized, as proposed earlier by Rego and Kirczenow [51] and discussed in more detail in the literature [52-54]. For studying dissipation on the microscopic level we realized highly doped suspended silicon nanowires with lateral dimensions down to $20 \mathrm{~nm}$ [50]. Due to random dopant fluctuations and segregation effects a potential landscape for electrons in the doped wire is created leading to a serial arrangement of Coulomb islands also termed multi-tunnel junctions (MTJs). These MTJs can then applied as Coulomb blockade thermometers (CBTs) [55-57] in suspended as well as non-suspended devices for comparison, allowing us to study dissipation in the low drain-source bias regime.

A strong modulation of the wire conductance found at high source-drain currents is related to heat transport by optical phonons. Since phonon generation depends on the phonon density 


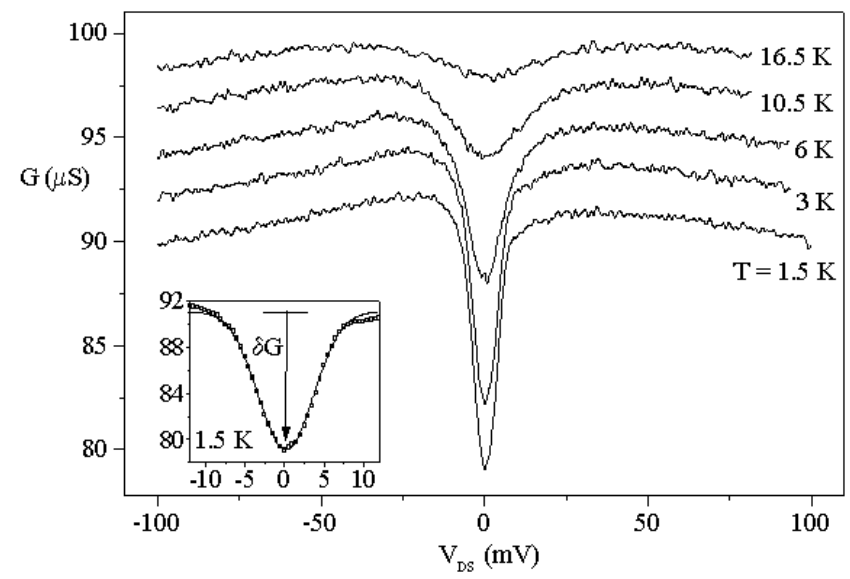

Figure 25. Conductance of a nanowire: at the lowest temperatures of $1.5 \mathrm{~K}$ a sharp resonance appears, resulting from MTJ in the wire. This temperature-dependent resonance around $V_{\mathrm{DS}}=0 \mathrm{~V}$ vanishes above $25 \mathrm{~K}$. The inset shows the conductance resonance at $1.5 \mathrm{~K}$ and a fit according to [11]. The resonance amplitude $\delta G$ is finally applied for thermometry.

of states at the temperature of the nanowire the phonon spectrum affects electron transport in the suspended nanostructures. The maximum current densities the nanowires can sustain is of the same order as for superconducting wires. Figure 26 shows the nonlinear $I V$-characteristic of a suspended nanowire as a function of temperature. We can conclude that internal dissipation has a lower limit defined by the effective electron-phonon relaxation mechanisms.

\subsection{Viscous or external losses}

When a resonator is immersed into a liquid or into a gaseous atmosphere the non-zero momentum transfer of the atoms effectively reduces the resonance amplitude [58-61]. Immersing a nanobeam in gaseous or fluid helium and inducing a mechanical vibration allows us to study the underlying principles of these external dissipation effects. In figure 27 the measurement of the reflected power during filling of the sample holder with ${ }^{4} \mathrm{He}$ is shown (the sample holder was cooled to $4.2 \mathrm{~K}$ ). The attenuation by the gas leads to a decrease of the resonance amplitude, a shift to lower frequencies and a broadening of the resonance. When liquefaction of ${ }^{4} \mathrm{He}$ occurs (shown in the last trace), the resonance disappears completely. As seen in this figure we find even in the linear regime of the resonator's response a dispersion when the ${ }^{4} \mathrm{He}$ content is increased. This corresponds to an effective momentum transfer to the ${ }^{4} \mathrm{He}$ atoms impinging onto the resonator. Increasing the power applied to the resonator, a non-zero amplitude of its motion immersed in the liquid is found.

It is quite interesting to use the nanoresonators for creating excitations in superfluid helium, since the velocity of the beam is of the same order compared to the critical velocity in ${ }^{4} \mathrm{He}[62]$. Until the beam reaches this velocity vortex line generation is considered to be low [3]. It has to be noted that losses into sound waves are still possible below the critical velocity. Further acceleration leads to the excitation of vortex states in the fluid resulting in an increased energy consumption. This can be seen in a flattening of the resonance curves. In ${ }^{3} \mathrm{He}$ the critical velocity is given by the energy required for pair breaking of the superfluid pairs, which is in the $\mathrm{mm} \mathrm{s}^{-1}$ range. In ${ }^{4} \mathrm{He}$ it is given by the creation of vortex states at low pressures and the excitation of rotons at higher pressures. The corresponding velocities for these excitations are about $25 \mathrm{~m} \mathrm{~s}^{-1}$. 

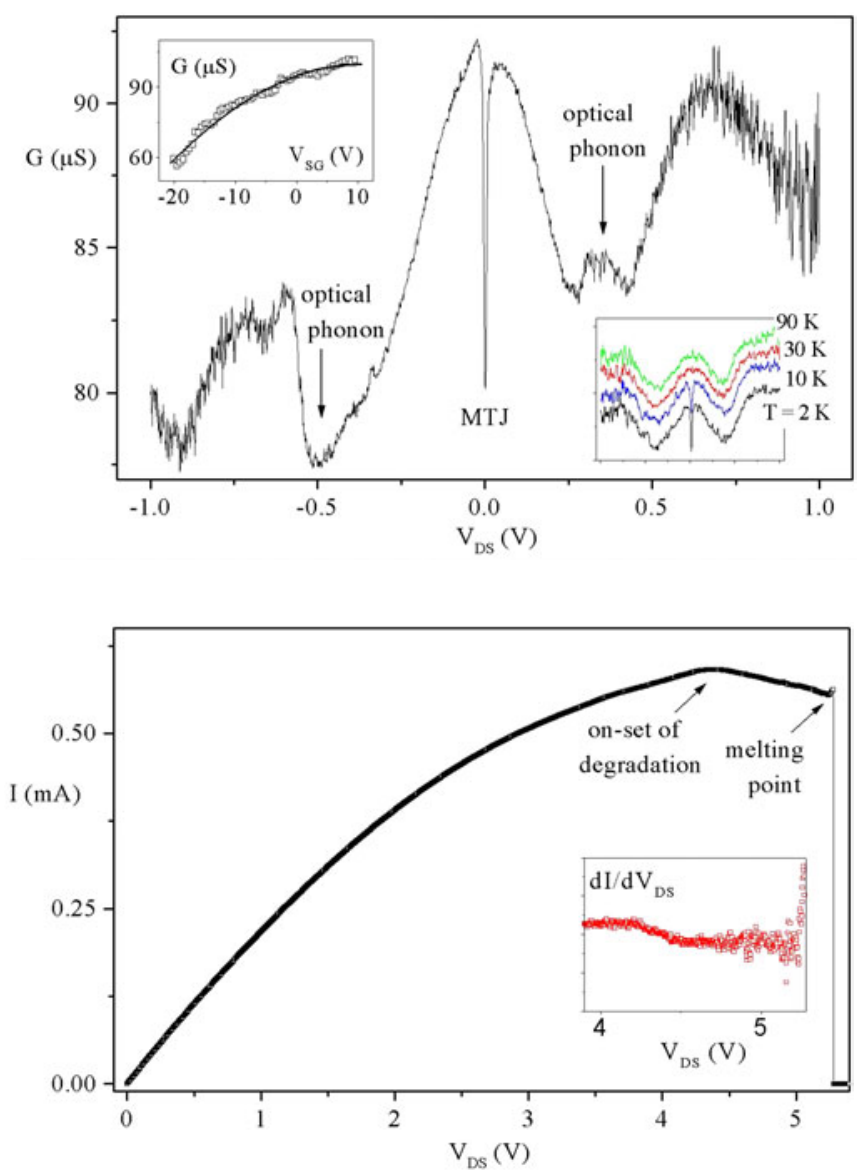

Figure 26. Top: conductance of the nanowire at high $V_{\mathrm{DS}}$. In the centre the MTJ resonance at $1.5 \mathrm{~K}$ is seen. Around $\pm 0.5 \mathrm{~V}$ we find a structure indicating electron relaxation via optical phonons. The lower right inset shows the insensitivity of the optical phonon relaxation peaks up to $90 \mathrm{~K}$. The upper left inset depicts the field effect for this particular suspended nanowire with the conductance as a function of side gate voltage $V_{\mathrm{SG}}$. Due to the strong doping the wire is not fully depleted. Bottom: maximum sustainable current through a $100 \times 140 \mathrm{~nm}^{2}$ thin suspended nanowire. The inset shows the conductance close to the melting point.

\subsection{Internal losses}

Another source of dissipation is given by the small size of the resonators, since the surface to volume ratio is quite different compared to macroscopic resonators. According to Carr et al [39] a reduction of $Q$ is found for larger surface to volume relation. In other words surface tension and in general surface defects and adsorbed layers (e.g. chemicals and water) are becoming increasingly important [63]. A detailed study will be presented elsewhere. Energy is also lost into the clamping points of the suspended nanostructures. Another dominant contribution to the losses is given by the so far used metallic top layer; i.e., the metal necessary to probe the resonators strongly enhances the overall mass of the system. However, the experimental data available suggest that surface defects and surface tension are the main cause for $Q$-factor reduction, since the dry etching process might introduce a number of defects. In figure 28 the nonlinear response of a mechanical resonator is shown: upon increasing the excitation power a hysteresis is observed with a substructure, which might be interpreted as an impurity state. 

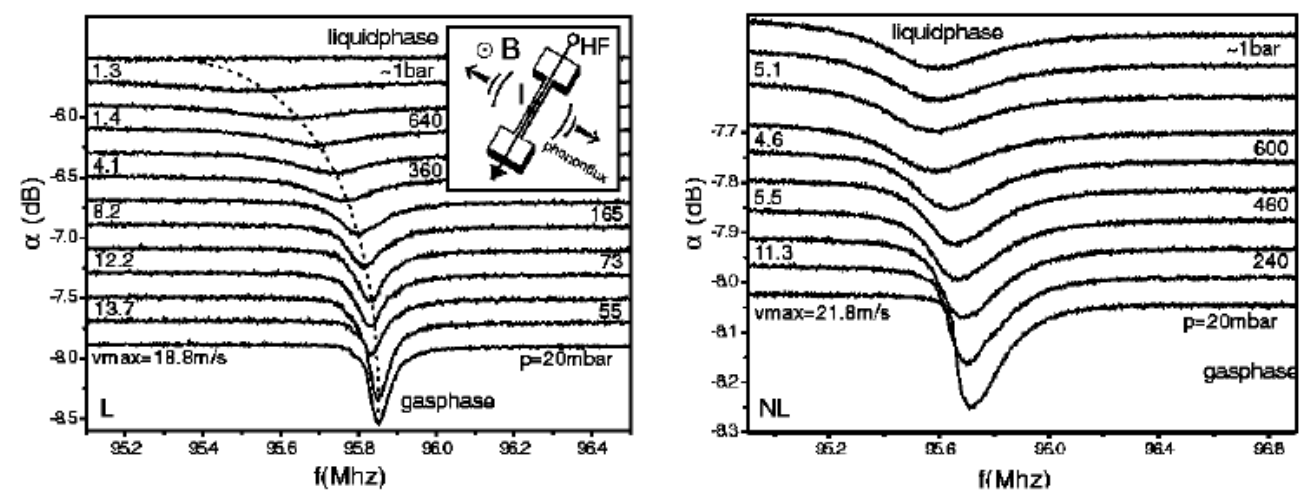

Figure 27. Attenuation of the mechanical resonance amplitude by increasing ${ }^{4} \mathrm{He}$ pressure as indicated until liquefaction occurs in the linear (left) and nonlinear (right) regime ( $-63 \mathrm{dBm}$ input power, $T=4.2 \mathrm{~K}$ ). The maximum velocity of the beam $v_{\max }$ has been calculated for each curve and is given on the left-hand side. In the inset a schematic view for directed phonon generation is sketched. The magnetic field is oriented perpendicular to the high frequency current $I$.

(a)

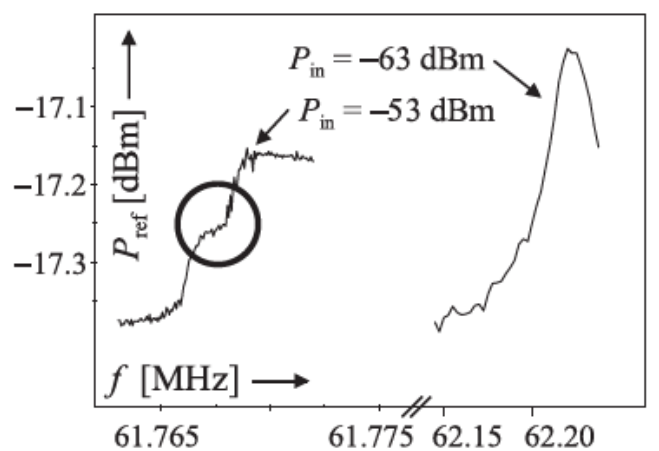

(b)

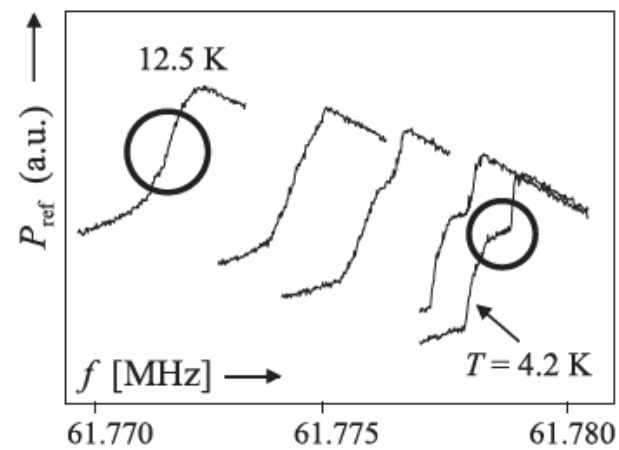

Figure 28. Spectra of a resonator: (a) the response of a nanobeam in the linear $\left(P_{\text {in }}=-63 \mathrm{dBm}\right)$ and nonlinear $\left(P_{\text {in }}=-53 \mathrm{dBm}\right)$ regime is depicted. The particular feature shown is the kink in the nonlinear trace, resulting from higher order nonlinearity. (b) This kink disappears upon increasing the sample's temperature to above $12 \mathrm{~K}$.

Such an impurity can either be an adsorbed monolayer of molecules on the beam's surface or an intrinsic impurity of the resonator. In both cases this leads to a higher order term in the potential, which in turn gives rise to a change in the restoring force. As seen in figure 28 this leads to an additional kink, which disappears towards higher temperatures. However, in detailed measurements applying different gate voltages in the close vicinity of the mechanical resonator we found that this specific kink results from a higher order nonlinearity introduced by the clamping points [9].

\subsection{Electronic environment}

The coupling of the mechanical circuit to the electronic probing circuit is well understood [64]: the mechanical oscillation in a magnetic field induces a voltage in the metallic beam, which is 
given by

$$
V(t)=\xi L B \dot{x}(t)=\mathrm{i} \omega \xi L B x(t),
$$

where $\xi$ is a constant of order unity and is determined by the specific mode. Without an external circuit connected, the voltage can be written

$$
V(\omega)=\begin{gathered}
\mathrm{i} \omega \xi L^{2} B^{2} / m_{\mathrm{eff}} \\
\omega_{0}^{2}-\omega^{2}+\mathrm{i} \omega \omega_{0} / Q_{0}
\end{gathered} I(\omega),
$$

where $Q_{0}=\omega_{0} / 2 \mu$ is the intrinsic $Q$-factor of the resonator. The magneto-motively driven resonator can be represented by a circuit with resistance $R_{\mathrm{m}}$, inductance $L_{\mathrm{m}}$ and capacitance $C_{\mathrm{m}}$ in parallel. The voltage drop across this circuit under the assumption of large external impedance $Z_{\mathrm{ext}} \rightarrow \infty$ is

$$
V(\omega)=\underset{\omega_{L C}^{2}-\omega^{2}+\mathrm{i} \omega / R_{\mathrm{m}} C_{\mathrm{m}}}{\mathrm{i} \omega / C_{\mathrm{m}}} I(\omega),
$$

where $\omega_{L C}=1 / \sqrt{ } L_{\mathrm{m}} C_{\mathrm{m}}$.

The two equations can be directly compared:

$$
C_{\mathrm{m}}=\begin{gathered}
m_{\mathrm{eff}} \\
\xi L^{2} B^{2}
\end{gathered}, \quad L_{\mathrm{m}}=\begin{aligned}
& \xi L^{2} B^{2} \\
& \omega_{0}^{2} m_{\mathrm{eff}}
\end{aligned}, \quad R_{\mathrm{m}}={ }_{\omega_{0} m_{\mathrm{eff}}}^{\xi L^{2} B^{2}} Q_{0} .
$$

This implies $\omega_{L C}=\omega_{0}$. We introduce now a finite external impedance under the assumption that $Z_{\text {ext }}(\omega)$ is constant when the mechanical resonance condition is met, which is satisfied when $Q_{0} \gg 1$. Approximating $Z_{\text {ext }}(\omega) \cong R_{\text {ext }}+\mathrm{i} X_{\text {ext }}$ the voltage drop is obtained

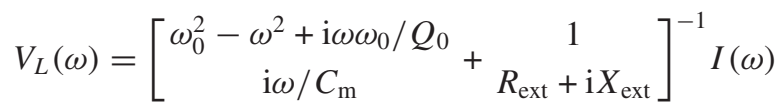

$$
\begin{aligned}
& ={ }_{\left(\omega_{0}^{2}+\omega \omega_{0} Z_{c} X_{\text {ext }} /\left|Z_{\text {ext }}\right|^{2}\right)-\omega^{2}+\mathrm{i} \omega \omega_{0}\left(1 / Q_{0}+Z_{c} R_{\text {ext }} /\left|Z_{\text {ext }}\right|^{2}\right)}^{I(\omega)}
\end{aligned}
$$

with the characteristic impedance $Z_{c}=\sqrt{ } L_{\mathrm{m}} / C_{\mathrm{m}}$. Obviously, the external impedance leads to a resonance shift and to a variation of $Q$. A series expansion with respect ${ }^{6}$ to $Z_{c} / Z_{\text {ext }}$ leads to

$$
f_{L}=f_{0} \sqrt{ } 1+Z_{c} X_{\mathrm{ext}} /\left|Z_{\mathrm{ext}}\right|^{2}
$$

and

$$
Q_{L}^{-1}=Q^{-1}+Z_{c} R_{\text {ext }} /\left|Z_{\text {ext }}\right|^{2} .
$$

Finally, it can be concluded that proper impedance matching of the external circuit (cable, gate structure etc) to a $50 \Omega$ load is necessary for building high- $Q$ mechanical resonators.

\section{Quantum-limited displacement detection}

One aim of building mechanical resonators on the nanometre scale is the possibility of accessing the realm of quantum mechanics [16]. The simple reasoning for this is the comparison of achievable mechanical energies of the system $\epsilon_{m}=h f_{0}$ and comparing this to the thermal energy of the connecting environment $\epsilon_{T}=k_{B} T$. For frequencies of the order of $0.5-1 \mathrm{GHz}$ the corresponding temperature is $50 \mathrm{mK}$, which is in the accessible range of dilution refrigerators. Theory work reflecting such an approach by Bocko and Onofrio [66] was based on classical scanning tunnelling microscopy tips suffering a finite back-action of the tunnelling electrons

6 This expansion is valid, since the resonators investigated commonly show $Z_{c}<0.01 \Omega$ and $Z_{\text {ext }}=50 \Omega$. 
onto the resonating clapper. Rugar and Grütter [36] then built a mechanical parametric amplifier for thermomechanical noise squeezing measurements, followed later by Greywall et al [28] and Yurke et al [29] who demonstrated effective noise reduction in nonlinear oscillators. The use of micromechanical devices for QND measurements was then investigated by Braginsky and Khalili [65] and Bocko and Onofrio [66].

The starting ground for QND measurements are commonly the observables of a harmonic oscillator. True QND observables require that they are decoupled from its conjugate observable, otherwise a measurement on the observable unavoidably would spoil the observable of interest. If such a measurement can be performed the mechanical resonator would be in a state where one observable is squeezed at the expense of the other [67]. This would greatly enhance the sensitivity of any mechanical system [68] and finally allow us to probe the decoherence of a macroscopic object [69]. An example calculation of such a squeezed state in a micron-sized cantilever was recently given by Blencowe and Wybourne [70, 71], showing the importance for scanning probe techniques.

Taking a vibrating wire of macroscopic dimensions, the wave equation describing its motion is essentially the same as for a mesoscopic mechanical resonator. The question naturally arising is how the two regimes can be easily identified. In general, the distinction between classical and quantum mechanics is given by the use of Heisenberg's uncertainty relation. There are different approaches which can be taken: the first one is realizing ultra-sensitive displacement detection by using tunnel sensors and quantum dots, relying on the relation $\Delta x \Delta p \sim \hbar$. The second approach is based on a more intricate method, i.e. on measuring the heat load of suspended nanocrystals and addressing the determination of a number state $(N)$ of the suspended system. This relies on the Heisenberg relation $\Delta N \Delta \phi \sim \hbar$. Recent measurements by Schwab et al [49] give strong evidence that indeed phonon quantization can be observed by using extremely sensitive SQUID noise thermometry. Extending this idea to QND measurements requires us to fix the resonator number or Fock state. This would allow us to count single phonons.

Following Bocko and Onofrio [66] a QND measurement on a harmonic oscillator can be described in terms of Hermitian coordinate $\hat{x}$ and momentum $\hat{p}$ operators. The mechanical system itself possesses a mass $m$ and resonates at an angular frequency $\omega$. Here, coupling to the environment via an interaction Hamiltonian $\hat{H}_{i}$ is neglected. The Hamiltonian of the system is then given by

$$
\hat{H}_{0}=\frac{\hat{p}}{2 m}+\begin{gathered}
m \omega^{2} \hat{x}^{2} \\
2
\end{gathered}
$$

while the number of phonons is given by the number operator

$$
\hat{n}=\frac{\hat{H}_{0}}{\hbar \omega}-\frac{1}{2}=\hat{a}^{\dagger} \hat{a}
$$

with the creation and annihilation operators

$$
\begin{aligned}
& \left.\hat{a}^{\dagger}=\sqrt{\frac{m \omega}{2 \hbar}(\hat{x}-\mathrm{i}} \begin{array}{c}
\hat{p} \\
m \omega
\end{array}\right), \\
& \left.\hat{a}=\sqrt{\frac{m \omega}{2 \hbar}(\hat{x}+\mathrm{i} \underset{m \omega}{m}}\right) .
\end{aligned}
$$

The frequency dependence of these operators can be simplified by defining the complex amplitudes $\hat{X}_{1}+\mathrm{i} \hat{X}_{2}$ to yield

$$
\hat{x}+\mathrm{i} \underset{m \omega}{\hat{p}}=\left(\hat{X}_{1}+\mathrm{i} \hat{X}_{2}\right) \exp (-\mathrm{i} \omega t) .
$$


This complex amplitude operator then is defined in terms of $\hat{x}$ and $\hat{p} / 2 \omega$ by splitting into the real and imaginary part:

$$
\begin{aligned}
& \hat{X}_{1}(\hat{x}, \hat{p}, t)=\hat{x} \cos \omega t-\hat{p}_{m \omega}^{\hat{p}} \sin \omega t, \\
& \hat{X}_{2}(\hat{x}, \hat{p}, t)=\hat{x} \sin \omega t+{ }_{m \omega}^{\hat{p}} \cos \omega t .
\end{aligned}
$$

In the absence of interactions these commute with themselves for all times $t$,

$$
\underset{\mathrm{d} t}{\mathrm{~d} \hat{X}_{j}}=\frac{\partial \hat{X}_{j}}{\partial t}-{ }_{\hbar}^{\mathrm{i}}\left[\hat{X}_{j}, \hat{H}_{0}\right]=0,
$$

which is the condition for a QND observable. For practical purposes it follows that the number of quanta $\hat{n}$ and the complex amplitudes $\hat{X}_{1}$ and $\hat{X}_{2}$ can be used as continuous QND observables.

In both of the above mentioned experimental approaches the question whether quantum mechanical systems can be realized is reduced to the degree of back-action of the detector onto the quantum device. The main problem is how to isolate the system from the environment, since any connection introduces decay channels leading to a destruction of coherence, i.e. a non-negligible interaction Hamiltonian. However, with $Q$-factors above $10^{3}$ nanomechanical systems are expected to reveal the transition to quantum mechanics at low enough temperatures. As we have seen in the earlier sections, it is indeed possible to build such freely suspended devices on the nanometre scale with eigenfrequencies approaching the $\mathrm{GHz}$ regime. Hence, it seems to be within reach to find a quantum mechanical response of a mechanical system, when the thermal coupling due to phonon quantization can be sufficiently reduced.

The question now is how to determine whether a mechanical oscillator can be regarded as a quantum mechanical object. Starting from the Duffing equation with the potential

$$
V_{0}(x)={ }_{2}^{1} m \omega^{2} x^{2}+{ }_{4}^{1} m \alpha x^{4},
$$

we consider typical values for the mechanical resonator: $m=6.15 \times 10^{-16} \mathrm{~kg}, \omega_{0}=2 \pi f_{0}=$ $2 \pi \cdot 74 \mathrm{MHz}=5 \times 10^{8} \mathrm{~Hz}$, for the anharmonicity $\alpha=9.1 \times 10^{28} \mathrm{~m} \mathrm{~s}^{-1}, \mu=50265 \mathrm{~Hz}$ and length $l=1.9 \mu \mathrm{m}, B=1 \mathrm{~T}$ and $I_{0}=19 \mu \mathrm{A}$. For the first scaling factor to be considered it is found (for $\alpha \rightarrow 0$ ) that

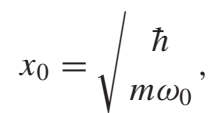

which yields $1.9 \times 10^{-5} \mathrm{~nm}$. Assuming achievable values of $m \sim 10^{-20} \mathrm{~kg}$ and $\omega=$ $1-2 \times 10^{9} \mathrm{~Hz}$ one obtains already $x_{0} \sim 3 \mathrm{pm}$. The energy of the lowest mode

$$
\epsilon_{0}=\hbar \omega_{0},
$$

is of the order of $\sim 10^{-34} \mathrm{~J} \mathrm{~s} \times 1 \mathrm{GHz} \cong 10^{-25} \mathrm{~J} \cong k_{B} \cdot 7.3 \mathrm{mK}$, a value which is just within reach of dilution refrigerators.

As noted before, the most reliable technique to probe nanomechanical resonators is recording the induced electromagnetic field by a magneto-motively driven beam. Detection can be performed by monitoring the reflected signal or-at least in principle - the transmitted power. Another possibility is given by tracing the displacement by a gate electrode coupling capacitively to the beam. In other words the displacement $\Delta x$ is translated into a variation of the capacitance $\Delta C_{\text {gate }}$. A possible back-action then should alter the beam's momentum $\Delta p$ and hence change the resonance pattern. This is not a QND measurement, since $\Delta x$ and $\Delta p$ are not Hermitian; such an experiment should allow us to probe the transition from classical mechanics into the realm of quantum mechanics. 

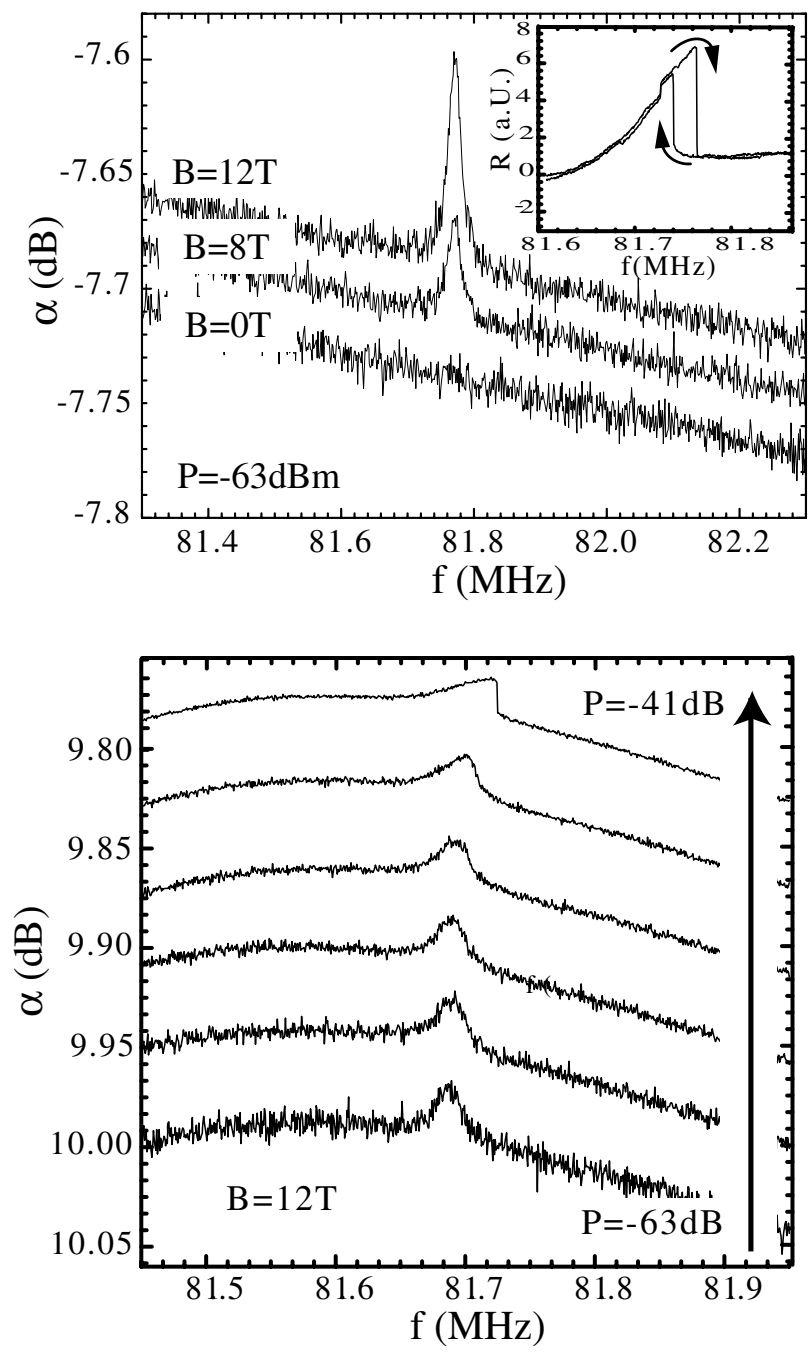

Figure 29. Top: measured resonances of a nanobeam: this mechanical resonator is $4.8 \mu \mathrm{m}$ long, $170 \mathrm{~nm}$ wide and $190 \mathrm{~nm}$ thick, covered by a $50 \mathrm{~nm}$ thick conducting Au layer. The magnetic field is oriented in the plane of the sample surface and perpendicular to the beam. The inset shows the observed hysteresis which emerges when tracing the frequency from low to high and the reverse. Bottom: resonances in the spectrum of a nanomechanical resonator detected by capacitive coupling.

The result when using capacitive detection to probe the resonances of a mechanical resonator is depicted in figure 29: in this measurement we observed $Q \sim 4150$ enhanced by a factor of 1.52 as compared to the value measured by the standard detection technique. As noted before the induced voltage over the beam due to its motion in the magnetic field can be written as

$$
V_{\text {ind }}(\omega)=\mathrm{i} \omega L B \xi u(\omega),
$$

where $\xi$ denotes a factor depending on the excited mode and $u$ the amplitude of the oscillation. This approach is justified if a periodic motion at frequency $\omega$ is assumed. In order to derive a similar equation for the case of capacitive detection it is of advantage to use an expansion to 


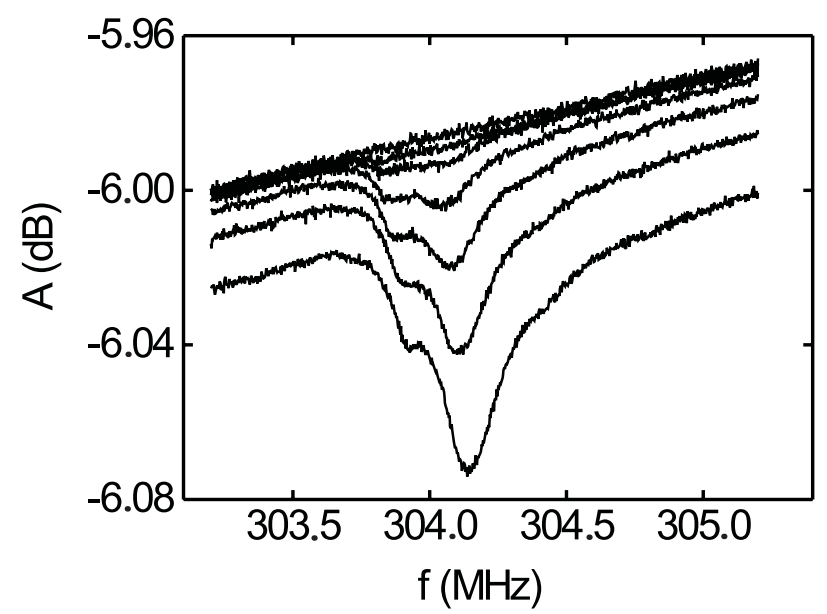

Figure 30. Highest frequencies measured for increasing magnetic fields.

the second order of the capacitance between the detection gate and the beam

$$
C(u)=C(0)+\begin{gathered}
\partial C(u) \\
\partial u
\end{gathered}\left|\begin{array}{ll}
1 & \partial^{2} C(u) \\
2 & \partial u^{2}
\end{array}\right|_{0} u^{2}+\cdots,
$$

where $C(u)$ denotes the dependence of the capacitance on the displacement of the beam perpendicular to the sample's surface. In equilibrium, the beam is in a state of minimum electrostatic energy, the first derivative of $C(u)$ with respect to the amplitude $u$ vanishes

$$
\left.\begin{gathered}
\partial C(u) \\
\partial u
\end{gathered}\right|_{0}=0
$$

and the induced voltage can be written, using equations (71) and (72), as

$$
V_{\text {cap }}(\omega)=\underset{C(u)}{Q}=V_{0}-\frac{1}{2} Q_{c} C_{0}^{2} C_{0}^{\prime \prime} u^{2}(\omega)
$$

where $C_{0}^{\prime \prime}$ denotes the second derivative of the capacitance with respect to the amplitude, taken at zero amplitude- $u(\omega)$ is assumed to have a Lorentzian shape

$$
u(\omega)=\frac{1}{\sqrt{\left(\omega_{0}^{2}-\omega^{2}\right)^{2}+4 \mu^{2} \omega^{2}}} .
$$

The different functional forms of equation (70), namely $\omega u(\omega)$, and of equation $(73),(u(\omega))^{2}$, result in two observable phenomena.

(i) The capacitively detected peaks are shifted by

$$
\Delta \omega=\omega_{0}-\sqrt{ } \omega_{0}^{2}-2 \mu^{2}
$$

whereby the maximum is situated at $\omega_{0}$ when detecting the reflected power.

(ii) As equation (73) depends on $u(\omega)^{2}$ the width of the resonance should be reduced when using capacitive detection. This results in an enhanced quality factor, depending on $\mu$ and $\omega$. 
The ratio of the quality factors for capacitive and direct detection can be calculated evaluating the full width at half maximum positions of equation (70) and equation (73) respectively and taking into account that in the experiment the power is measured and not a voltage. This ratio $\Lambda$ is a function of the resonance frequency $\omega_{0}$ and of the effective attenuation constant $\mu$. Using the experimentally found value of $\mu=94.2 \times 10^{3} \mathrm{~s}^{-1}, \Lambda=1.55$ is obtained, which agrees very well with the measured value of 1.52 . To summarize, we have shown that capacitive detection of NEMS displacement can be integrated as a highly sensitive probing mechanism. Apart from implementing the detection technique another straightforward approach to further increase the operating frequency (see figure 30) and force sensitivity of NEMS is to pump the nanomechanical system on the fundamental mode while probing capacitively on one of the harmonic modes. Applying parametrical amplification for QND measurements will further improve resolution [72].

\section{Outlook}

Three-dimensional nanostructuring of silicon was applied to fabricate mechanical devices on the nanometre scale. The properties of these structures are probed by RF techniques. Unprecedented mechanical resonance frequencies of the order of $350 \mathrm{MHz}$ were found. Applications in sensor technology were demonstrated, promising integration in fast and highly sensitive circuitry. The mechanical degree of freedom allows study of nonlinear dynamics on the nanometre scale and possibly reaching the quantum regime at temperatures below $100 \mathrm{mK}$. This is of great importance not only for the ultimate resolution of scanning probe microscopy, but also for quantum non-demolition measurements.

As an outlook one might consider the application of NEMS as mechanical memory elements. This idea dates back into the 19th century when Charles Babbage invented the first mechanical computing machines. These were clumsy and hardly ever operational. Nevertheless, nowadays the increasing integration density, the combination with electronic components and the high speed of operation lead to a certain renaissance of mechanical components. First realizations of mechanical transducers as memory elements have been given already by Hälg [75]. Other work used fullerenes caged in short nanotubes as mechanical memory cells [76]. Carbon nanotubes are of great interest regarding their mechanical properties [77, 78]. Moreover, the insensitivity against electromagnetic pulses and radiation, the low power consumption, the low defect density and usage of standard silicon processing techniques will support such ideas.

Another path to be followed is probing and connecting molecules [79], and cells [80], to mechanical semiconductor devices with $\AA$ resolution. The next few years will witness the development of the chemistry required and the proper theory to describe contacts between artificially fabricated systems and appropriate molecules. Yet one of the most promising topics that nanomechanical tools allow us to access is the field of bio-NEMS. The starting point for these investigations is the ability to design and build semiconductor devices on the length scale of $10 \mathrm{~nm}$, the same size as large proteins. This might ultimately lead to the integration of molecular motors [81] into NEMS as pioneered by Montemagno and Bachand [82].

\section{Acknowledgments}

This work has been supported by the Deutsche Forschungsgemeinschaft (DFG projects B1/4871 and Bl/487-2), the Bundesministerium für Forschung und Technologie (BMBF project Silizium-Nanoelektronik), the Defense Advanced Research Projects Agency (DARPA project 
Ultra), J P Kotthaus through the Max Planck Award, the Alexander von Humboldt society (follow-up funds to the Feodor Lynen Award), the Max Planck Society (Otto Hahn Award during the early stages) and Nano-Tools (project plow-01). RHB would like to thank Armin Kriele, Armin Tilke, Stephan Manus and Kim Harris for help during the course of this project.

\section{References}

[1] Irmer B, Blick R H, Simmel F, Gödel W, Lorenz H and Kotthaus J P 1998 Josephson junctions defined by a nanoplow Appl. Phys. Lett. 732051

Irmer B 1999 Nano-lithographie mit dem rasterkraftmikroskop PhD Thesis Ludwig-Maximilians-Universität, München

[2] Hendrix J M 1967 Jimi Hendrix Experience (Radio One)

[3] Landau L D and Lifschitz E M 1987 Theoretische Physik, Bd. I Mechanik vol 12 (Berlin: Akademie)

[4] Kinsler L E, Frey A R, Coppens A B and Sanders J V 2000 Fundamentals of Acoustics (New York: Wiley)

[5] Nayfeh A H and Mook D T 1995 Nonlinear Oscillations (New York: Wiley)

[6] Tilmans H A C, Elwenspoek M and Fluitman J H J 1992 Nonlinearity and hysteresis of resonant strain gauges Sensors Actuators A 3035

[7] Magnus K and Popp K 1997 Schwingungen (Stuttgart: Teubner)

[8] Padua A A H, Faraleira J M N A, Calado J C G and Wakeham W A 1998 Electromechanical model for vibratingwire instruments Rev. Sci. Instrum. 692393

[9] Scheible D V 2001 In-situ verstimmbare nanomechanische Resonatoren Diploma Thesis Ludwig-MaximiliansUniversität, München

Scheible D V, Erbe A, Blick R H and Corso G 2002 Appl. Phys. Lett. at press

[10] Erbe A, Weiss Ch, Zwerger W and Blick R H 2001 An electro-mechanical single electron transistor Phys. Rev. Lett. 87096106

[11] Colinge J-P 1991 Silicon-on-Insulator Technology: Materials to VLSI (Boston, MA: Kluwer)

[12] Bruel M 1993 FR Patent Specification 2681472

Bruel M 1994 US Patent Specification 5374564

[13] Elwenspoek M and Jansen H V 1998 Silicon Micromachining (Cambridge: Cambridge University Press)

[14] Pescini L 1999 Suspended nanostructures in silicon-on-insulator materials Diploma Thesis Università degli Studi di Firenze and Ludwig-Maximilians-Universität, München

[15] Pescini L, Tilke A, Blick R H, Lorenz H, Kotthaus J P, Eberhardt W and Kern D 1999 Suspending highly doped silicon-on-insulator wires for applications in nanomechanics Nanotechnology 10418

[16] Blick R H, Erbe A, Tilke A and Wixforth A 2000 Auf dem Weg zur 'Quanten-Mechanik' Phys. Bl. 131

[17] Tilke A, Rotter M, Blick R H, Lorenz H and Kotthaus J P 2000 Single-crystalline silicon lift-off films for metal oxide semiconductor devices on arbitrary substrates Appl. Phys. Lett. 77558

[18] Cleland A N and Roukes M L 1996 Fabrication of high frequency nanometer scale mechanical resonators from bulk Si crystals Appl. Phys. Lett. 692653

[19] Krömmer H 1999 Nanomechanische Resonatoren auf der Grundlage von freitragenden Gold-SiliziumHybridstrukturen Diploma Thesis Ludwig-Maximilians-Universität, München

[20] Krömmer H, Erbe A, Tilke A, Manus S M and Blick R H 2000 Nanomechanical resonators operating as charge detectors in the nonlinear regime Europhys. Lett. 50101

[21] Carr D W, Evoy S, Sekaric L, Craighead H G and Parpia J M 1999 Measurement of mechanical resonance and losses in nanometer scale silicon wires Appl. Phys. Lett. 75920

[22] Duh K H G, Pospieszalski M W, Kopp W F, Ho P, Jabra A A, Chao P-C, Smith P M, Lester L F, Ballingall J M and Weinreb S 1988 Ultra-low-noise cryogenic high-electron-mobility transistors IEEE Trans. Electron. Device 35249

[23] Cleland A N and Roukes M L 1998 A nanometre-scale mechanical electrometer Nature 392160

[24] Kraus A 2000 Getriebene nichtlineare nanomechanische Resonatoren Diploma Thesis Ludwig-MaximiliansUniversität, München

[25] Beil F W, Pescini L, Hoehberger E, Kraus A, Erbe A and Blick R H 2002 Comparing schemes of displacement detection in nanomachined mechanical resonators and subharmonic generation IEEE J. Micromech. Devices (Preprint cond-mat/0011299)

[26] Blick R H, Erbe A, Tilke A, Krömmer H, Pescini L, Manus S, Kriel A and Kotthaus J P 1999 Nanomechanical resonators operating in the radio frequency regime as single charge detectors Adv. Solid State Phys. 39121

[27] Nguyen N M and Meyer R G 1990 Microelectromechanical systems for radio frequency applications IEEE J. Solid-State Circuits 251028 
Maluf N 2000 An Introduction to Microelectromechanical Systems Engineering (Microelectromechanical Systems Series) (Norwood, MA: Artech) p 02062

de los Santos H J 1999 Introduction to microelectromechanical microwave systems (Microelectromechanical Systems Series) (Norwood, MA: Artech) p 02062

Nguyen C T-C 1999 Microelectromechanical components for miniaturized low-power communications Preprint

[28] Greywall D S, Yurke B, Busch P A, Pargellis A N and Willett R L 1994 Evading amplifier noise in nonlinear oscillators Phys. Rev. Lett. 722992

[29] Yurke B, Greywall D S, Pargellis A N and Busch P A 1995 Theory of amplifier-noise evasion in an oscillator employing a nonlinear resonator Phys. Rev. A 514211

[30] Schäffler F 1994 Strained Si/SiGe heterostructures for device applications Solid State Electron. 37765

Lee S J, Khurgin J B, Wang K L, Alt K, Ehrlich M J and Wagner J W 1997 Pressure and strain sensors based on intervalley electron transfer in AlGaAs Appl. Phys. Lett. 703437

Rego L G C and Kirczenow G 1999 Electrostatic mechanism for cooling semiconductor heterostructures Appl. Phys. Lett. 752262

Schmidt O G and Eberl K 2001 Revoluting layers in nanotechnology Nature 410168

[31] Pechmann R, Köhler J M, Fritzsche W, Schaper A and Jovin T M 1994 The Novolever: a new cantilever for scanning force microscopy microfabricated from polymeric materials Rev. Sci. Instrum. 653205

[32] Parlitz U and Lauterborn W 1985 Phys. Lett. A 10735

[33] Erbe A, Corso G, Krömmer H, Kraus A, Richter K and Blick R H 2000 Mechanical mixing in nonlinear nanomechanical resonators Appl. Phys. Lett. 773102 (Preprint cond-mat/9912278)

[34] Aime J P, Boisgard R, Nony L and Couturier G 1999 Nonlinear dynamic behaviour of an oscillating tipmicrolever system and contrast at the atomic scale Phys. Rev. Lett. 823388

[35] Isacsson A 2001 Dynamics of a three-terminal mechanically flexible tunneling contact Phys. Rev. B 64035326

[36] Rugar D and Grütter P 1991 Mechanical parametric amplification and thermomechanical noise squeezing Phys. Rev. Lett. 67699

[37] Turner K L, Miller S A, Hartwell P G, MacDonald N C, Strogatz S H and Adams S G 1998 Five parametric resonances in a microelectromechanical system Nature 396149

[38] Dana A, Ho F and Yamamoto Y 1998 Mechanical parametric amplification in piezoresistive gallium arsenide microcantilevers Appl. Phys. Lett. 721152

[39] Carr D W, Evoy S, Sekaric L, Craighead H G and Parpia J M 2000 Parametric amplification in a torsional microresonator Appl. Phys. Lett. 771545

[40] Kraus A, Erbe A, Corso G, Richter K and Blick R H 2001 Parametric frequency tuning of a phase locked nano-electromechanical resonator Appl. Phys. Lett. 793521

[41] Magde D and Mahr H 1967 Study in ammonium dihydrogen phosphate of spontaneous parametric interaction tunable from 4400 to $16000 \AA$ A Phys. Rev. Lett. 18905

[42] Lifshitz R and Roukes M L 2000 Thermoelastic damping in micro-and nano-mechanical systems Phys. Rev. B 615600

[43] Braginsky V B, Mitrofanov V P and Panov V I 1985 Systems with Small Dissipation (Chicago, IL: University of Chicago Press

[44] Tamayo J 1999 Energy dissipation in tapping-mode scanning force microscopy with low quality factors Appl. Phys. Lett. 753569

[45] Duffy W 1990 Acoustic quality factor of aluminium alloys from $50 \mathrm{mK}$ to $300 \mathrm{~K}$ J. Appl. Phys. 685601

[46] Kaganov M I, Lifshitz I M and Tanatarov L V 1957 Relaxation between electrons and the crystalline lattice Sov. Phys.-JETP 4173

[47] Bockelmann U and Bastard G 1990 Phonon scattering and energy relaxation in two-, one-, and zero-dimensional electron gases Phys. Rev. B 428947

[48] Fujii H, Kanemaru S, Matsukawa T and Itoh J 1999 Air-bridge-structured silicon nanowire and anomalous conductivity Appl. Phys. Lett. 753986

[49] Schwab K, Henriksen E A, Worlock J M and Roukes M L 2000 Measurement of the quantum of thermal conductance Nature 404974

[50] Tilke A, Pescini L, Erbe A, Lorenz H and Blick R H 2002 Phonon-mediated dissipation in suspended phosphorusdoped silicon nanowires Nanotechnology 13491

[51] Rego L G C and Kirczenow G 1998 Quantized thermal conductance of dielectric quantum wires Phys. Rev. Lett. 81232

[52] Roukes M L 1999 Yoctocalorimetry: phonon counting in nanostructures Physica B 263/264 1

[53] Angelescu D E, Cross M C and Roukes M L 1998 Heat transport in mesoscopic systems Superlatt. Microstruct. 23673 
[54] Greywall D S, Yurke B, Busch P A and Arney S C 1996 Low-temperature anomalies in the dissipation of small mechanical resonators Europhys. Lett. 3437

[55] Pekola J P, Kauppinen J P and Paalanen M A 1994 Thermometry by arrays of tunnel junctions Phys. Rev. Lett. 732903

[56] Pekola J P, Taskinen L J and Farhangfar Sh 2000 One- and two-dimensional tunnel junction arrays in weak Coulomb blockade regime: absolute accuracy in thermometry Appl. Phys. Lett. 763747

[57] DiTusa J F, Lin K, Park M, Isaacson M S and Parpia J M 1992 Role of phonon dimensionality on electron-phonon scattering rates Phys. Rev. Lett. 681156

[58] Weigert S, Dreier M and Hegner M 1996 Frequency shifts of cantilevers vibrating in various media Appl. Phys. Lett. 692834

[59] Leveque G, Girard P, Belaidi S and Cohen Solal G 1997 Effects of air damping in noncontact resonant force microscopy Rev. Sci. Instrum. 684137

[60] Kraus A, Erbe A and Blick R H 2000 Nanomechanical vibrating wire resonator for phonon spectroscopy in helium Nanotechnology 113

[61] Chon J W M, Mulvaney P and Sader J E 2000 Experimental validation of theoretical models for the frequency response of atomic force microscope cantilever beams immersed in fluids J. Appl. Phys. 873978

[62] Tilley D R and Tilley J 1990 Superfluidity and Superconductivity (New York: Hilger)

[63] Maboudian R and Howe R T 1997 Critical review: adhesion in surface micromachining structures J. Vac. Sci. Technol. B 151

[64] Cleland A N and Roukes M L 1999 External control of dissipation in a nanometer-scale radio frequency mechanical resonator Sensors Actuators A 72256

[65] Braginsky V B and Khalili F Ya 1996 Quantum nondemolition measurements: the route from toys to tools Rev. Mod. Phys. 681

[66] Bocko M F and Onofrio R 1996 On the measurement of a weak classical force coupled to a harmonic oscillator: experimental progress Rev. Mod. Phys. 68755

[67] Hu X and Nori F 1996 Squeezed phonon states: modulating quantum fluctuations of atomic displacement Phys. Rev. Lett. 762294

[68] Saulson P R 1990 Thermal noise in mechanical experiments Phys. Rev. D 422437

[69] Bose S, Jacobs K and Knight P L 1999 Scheme to probe the decoherence of a macroscopic object Phys. Rev. A 593204

[70] Blencowe M P and Wybourne M N 2000 Quantum squeezing of mechanical motion for micro-sized cantilevers Physica B 280555

[71] Blencowe M P and Wybourne M N 2000 Sensitivity of a micromechanical displacement detector based on the radio-frequency single-electron transistor Appl. Phys. Lett. 773845

[72] Gagen M J and Milburn G J 1991 Quantum nondemolition measurements using a fully parametric interaction Phys. Rev. A 436177

[73] Jager E W H, Smela E and Inganäs O 2000 Microfabricating conjugated polymer actuators Science 2901540

[74] Whaley S R, English D S, Hu E L, Barbara P F and Belcher A M 2000 Selection of peptides with semiconductor binding specificity for directed nanocrystal assembly Nature $\mathbf{4 0 5} 665$

[75] Hälg B 1990 On a nonvolatile memory cell based on micro-electro-mechanics IEEE Trans. Electron. Device 4 172

[76] Kwon Y-K, Tomanek D and Iijima S 1999 Bucky shuttle memory device: synthetic approach and molecular dynamics simulations Phys. Rev. Lett. 821470

[77] Yakobson B I, Brabec C J and Bernholc J 1996 Nanomechanics of carbon tubes: instabilities beyond linear response Phys. Rev. Lett. 762511

[78] Reulet B, Kasumov A Yu, Kociak M, Deblock R, Khodos I I, Gorbatov Yu B, Volkov V T, Journet C and Bouchiat H 2000 Acoustoelectric effect in carbon nanotubes Phys. Rev. Lett. 852829

[79] Fritz J, Baller M K, Lang H P, Rothuizen H, Vettiger P, Meyer E, Güntherodt H-J, Gerber Ch and Gimzewski J K 2000 Translating biomolecular recognition into nanomechanics Science 288316

[80] Ilic B, Czaplewski D, Craighead H G, Neuzil P, Campagnolo C and Batt C 2000 Mechanical resonant immunospecific biological detector Appl. Phys. Lett. 77450

[81] Howard J 1997 Molecular motors: structural adaptions to cellular functions Nature 389561

Kitamaru K, Tokunaga M, Iwane A H and Yanagida T 1999 A single myosin head moves along an actin filament with regular steps of $5.3 \mathrm{~nm}$ Nature 397129

Limberis L and Stewart R J 2000 Toward kinesin-powered microdevices Nanotechnology 1147

[82] Montemagno C and Bachand G 1999 Constructing nanomechanical devices powered by biomolecular motors Nanotechnology 10225 